\title{
A tritrophic interaction model for an olive tree pest, the olive moth - Prays oleae (Bernard)
}

\author{
Sonia Pappalardo ${ }^{\mathrm{a}}$, María Villa ${ }^{\mathrm{b}}$, Sónia A.P. Santos ${ }^{\mathrm{c}, \mathrm{d}}$, Jacinto Benhadi-Marín ${ }^{\mathrm{b}}$, \\ José Alberto Pereira ${ }^{\mathrm{b}}$, Ezio Venturino ${ }^{\mathrm{a}, *, 1}$ \\ a Dipartimento di Matematica “Giuseppe Peano", via Carlo Alberto 10, Università di Torino, 10123 Torino, Italy \\ ${ }^{\mathrm{b}}$ Centro de Investigação de Montanha (CIMO), Instituto Politécnico de Bragança, Campus de Santa Apolónia, 5300-253 Bragança, Portugal \\ c CIQuiBio, Barreiro School of Technology, Polytechnic Institute of Setúbal, Rua Américo da Silva Marinha, 2839-001 Lavradio, Portugal \\ ${ }^{\mathrm{d}}$ LEAF-Linking Landscape, Environment, Agriculture and Food, Instituto Superior de Agronomia, Universidade de Lisboa, Tapada da \\ Ajuda, 1349-017 Lisboa, Portugal
}

\section{A R T I C L E I N F O}

\section{MSC:}

$92 \mathrm{D} 25$

$92 \mathrm{D} 40$

Keywords:

Biological control

Pest control

Parasitoids

Predators, Modeling

Dynamical systems

Predator-prey

Moth

Spiders

\begin{abstract}
A B S T R A C T
The olive tree (Olea europaea L.) is among the oldest and most widespread crops in the Mediterranean basin. Portugal is the third olive producer in the European Union, and Trás-os-Montes region, located in northeastern Portugal, is the second Portuguese producing olive region.

The olive moth, Prays oleae (Bernard) (Lepidoptera: Praydidae) is a key olive pest in Trás-os-Montes. This pest is a natural host/prey of several organisms which include larvae of generalist and specialist parasitoids as well as generalist predators and entomopathogens. Its most abundant parasitoid is the specialist Ageniaspis fuscicollis (Dalman) (Hymenoptera: Encyrtidae) and this, in Trás-os-Montes region, is commonly followed by the facultative hyperparasitoid Elasmus flabellatus (Fonscolombe) (Hymenoptera: Eulophidae). Spiders represent a relevant group of generalist predators in olive agroecosystems and encompass an important predatory action in agroecosystems as well as an ability to reduce the populations of various insect pests. In this context, a mathematical model, considering the population of the olive moth, the two parasitoids populations and the spider population as the variables in our system, was constructed. The ecosystem steady states for feasibility and stability were assessed. The possible pesticide effects, that represent essentially extra mortality rates for each one of the insect populations, and potential abundance variations on their populations under a climate change scenario were included. Results indicate that the most important natural control agent is $A$. fuscicollis but in certain conditions E. flabellatus or spiders may be relevant contributors for the pest reduction. This approach may provide a useful tool to assist the field researchers on this pest system and its management.
\end{abstract}

\section{Introduction}

The olive tree (Olea europaea L.), one of the oldest and most widespread crops, has characterized economically, socially and culturally the populations of the Mediterranean basin. Nowadays, it is cultivated in all regions with climatic conditions that allow its establishment (Bartolini and Petruccelli, 2002). Portugal is one important olive producer country and Trás-os-Montes region, located in northeastern Portugal, in 2019 was responsible for the production of 914504 tons of olives in 359949 ha of groves (INE, 2021).

However, the olive tree is attacked by several pests that result in severe economical losses. The olive moth, Prays oleae (Bernard) (Lepidoptera: Praydidae) is the most damaging pest in Trás-os-Montes.
The insect has three generations a year and their larval stages attack different organs of the olive tree. Eggs of the anthophagous generation are laid on flower buds and, after hatching, larvae feed on flowers. Its adult flight period occurs at the end of the spring, laying the eggs of the carpophagous generation on the olive calyx. The carpophagous generation larvae bore into the olive stone and feed on the seed. At the end of the summer and beginning of the autumn, adults emerge and lay the phyllophagous generation eggs on the olive leaves. The phyllophagous larvae feed on the leaves and dig tunnels into them, where they overwinter until the following spring (Arambourg and Pralavorio, 1986).

\footnotetext{
* Corresponding author.

E-mail addresses: soniapappalardo96@gmail.com (S. Pappalardo), mariavilla@ipb.pt (M. Villa), Sonia.Santos@estbarreiro.ips.pt (S.A.P. Santos), jbenma@ipb.pt (J. Benhadi-Marín),jpereira@ipb.pt (J.A. Pereira), ezio.venturino@unito.it (E. Venturino).

1 Member of the INDAM research group GNCS.
} 
P. oleae is naturally controlled by several organisms which include several generalist and specialist parasitoids as well as generalist predators. The most abundant is the parasitoid Ageniaspis fuscicollis (Dalman) (Hymenoptera: Encyrtidae) (Bento et al., 1998; Herz et al., 2005; Villa et al., 2016a). This specialist parasitoid of the olive moth is well synchronized phenologically with the pest (Campos and Ramos, 1982). In Trás-os-Montes region, in some years the second most abundant parasitoid was Elasmus flabellatus (Fonscolombe) (Hymenoptera: Eulophidae) (Villa et al., 2016a). It behaves as a facultative hyperparasitoid, parasitizing some larvae of hymenopteran and larvae and pupae of lepidopteran species (Yefremova and Strakhova, 2010) and references therein. Adult parasitoids need energy for maintenance, locomotion and reproduction that is provided by non-host foods such as flowers and insect honeydews (Jervis et al., 2008, 1993). In Trásos-Montes olive groves, honeydews produced by some secondary pests, such as the black scale, Saissetia oleae (Olivier) (Hemiptera: Coccidae) and the olive psyllid Euphyllura olivina (Costa) (Hemiptera: Psyllidae) and flowers from many plant species within and around the olive groves probably nourish the olive moth parasitoids.

The olive moth is also attacked by generalist predators. Spiders are generalist predators with important predatory action in agroecosystems and ability to reduce the populations of various insect pests (Marc et al., 1999; Nyffeler and Sunderland, 2003; Riechert and Lockley, 1984). In this context, some spiders exhibit a high degree of superfluous killing (individuals attack more prey that they actually consume) at high levels of prey density (Riechert and Maupin, 1997). Spiders constitute one of the most abundant group of predators in the olive agroecosystem and have ubiquitous feeding habits (Pascual et al., 2010; Benhadi-Marín et al., 2016). During the spring and coinciding with the adult flight of the anthophagous generation, several abundant spiders in the olive canopy potentially prey on the olive moth (Pascual et al., 2010). In addition, it is of paramount importance to understand the behavior of this type of tritrophic relations under a climate change context because shifts in plant and insect phenologies, distribution or voltinism might result in modification of the trophic interactions timing and distribution (Castex et al., 2018). Our goal is to develop a mathematical model to generate population behavioral predictions under different abundance scenarios, resulting from the potential effects of climate change, for the various agents involved in the trophic system under consideration (the pest- $P$. oleae, its main parasitoids, $-A$. fuscicollis and E. flabellatus, and spiders - as model for an abundant generalist predator in the olive tree canopy).

\section{Materials and methods}

We consider the olive moth $M$ population, the E. flabellatus population $E$, the $A$. fuscicollis population $A$ and the spiders population $S$ as the variables in our system.

The spiders feed on the adult populations of the two parasitoids and on the olive moth. Their hunting rate on $E$ is $u$, the one on $A$ is $v$ and the one on $M$ is $w$, with respective conversion coefficients $q_{i}$, $i \in\{E, A, M\}$. But since they are generalist predators, they have also other resources modeled in a logistic fashion with carrying capacity $L$ (i.e. the theoretical amount of individuals of the population that the environmental conditions can sustain) and net reproduction rate $z$ (i.e. the difference between reproduction rate and mortality rate), described by the last term. Their dynamics is expressed in the first equation of the system.

The second equation describes the development of the parasitoid $E$. flabellatus, $E$. They feed by parasitizing either the other parasitoid $A$, at rate $a$, with conversion coefficient $e$,or $M$, the moth, at rate $b$ with conversion coefficient $h$. Consequently they have the resources to reproduce. The first two terms in the second equation describe jointly the parasitizing and reproduction processes, exerted on both prey. In addition they thrive also by feeding on sugary liquids (nectar or honeydews) or parasitizing other insects, that are not explicitly modeled.
This gives the additional third logistic term with net reproduction rate $r$ and intraspecific competition rate $r K^{-1}$, where $K$ represents the carrying capacity of these extra resources. The last two terms represent mortalities induced by spiders hunting or use of pesticides.

The third equation contains the dynamics of the adult individuals of the parasitoid $A$. fuscicollis, $A$. They reproduce at rate $c$ by feeding on the moth $M$, with conversion coefficient $g$ and they feed also on sugary liquids in the environment (honeydews or nectar) that, as we said before, we do not include in the model. Sugary liquids would improve the reproduction but $A$. fuscicollis can survive without them while they cannot survive without the moth. This is because reproduction depends strongly on the moth (being specialist parasitoids, they do not parasitize other insects). Thus, if the moths vanish, even if there are sugary liquids in the environment, $A$. fuscicollis cannot reproduce, but if the sugary liquids are absent, the moths can still reproduce. This is modeled by the parameter $g$, which can be thought to be split among a baseline value $g_{0}$ and a boost term $g_{1}$ provided by the possible presence of sugary liquids. Thus: $g=g_{0}+g_{1}$. In addition, we introduce $f$, the rate of intraspecific competition of $A$, the natural mortality $m_{A}$ and hunting by both $E$ and $S$ at respective rates $a$ and $v$ (as seen above).

The olive moth $M$, last equation, is attacked by both parasitoids larvae $E$ and $A$, as well as $S$, at respective rates $b, c$ and $w$. The moth logistic exploitation of the resources is modeled by the fourth term, where $U$ denotes the carrying capacity represented by the olive crop and $s$ is net reproduction rate.

In all equations, we include also the possible pesticide effects, that represent extra mortality rates for each populations, $k_{i}, i \in$ $\{S, E, A, M\}$. They appear in the system as the last terms of each equation.

The resulting system, where all the parameters are nonnegative and whose interpretation is given in Table Table 1, reads:

$$
\begin{aligned}
\frac{d S}{d t}= & \left(u q_{E} E+v q_{A} A+w q_{M} M\right) S \\
& +z S\left(1-\frac{S}{L}\right)-k_{S} S, \\
\frac{d E}{d t}= & e a E A+h b E M \\
& +r E\left(1-\frac{E}{K}\right)-u E S-k_{E} E, \\
\frac{d A}{d t}= & g c A M-m_{A} A-f A^{2} \\
& -a E A-v A S-k_{A} A, \\
\frac{d M}{d t}= & -b E M-c A M-w M S \\
& +s M\left(1-\frac{M}{U}\right)-k_{M} M .
\end{aligned}
$$

\section{Results}

\subsection{Analysis of the equilibrium points}

In this section we analyze all the possible equilibrium points of the system (2.1), for both feasibility and stability, deferring to Appendix A the more complicated mathematical details.

\subsubsection{Feasibility}

At first, the origin $P_{0}$ is a feasible equilibrium. Then there are the points with just one nonvanishing population, $P_{1}=\left(S_{1}, 0,0,0\right), P_{2}=$ $\left(0, E_{2}, 0,0\right)$ and $P_{8}=\left(0,0,0, M_{8}\right)$, with population values and feasibility condition respectively given by

$$
\begin{array}{cl}
S_{1}=\frac{L\left(z-k_{S}\right)}{z}, & z>k_{S}, \\
E_{2}=\frac{K\left(r-k_{E}\right)}{r}, & r>k_{E}, \\
M_{8}=\frac{U\left(s-k_{M}\right)}{s}, & s>k_{M} .
\end{array}
$$


Table 1

The parameters and their interpretation.

\begin{tabular}{ll}
\hline Parameter & Biological interpretation \\
\hline$u$ & Spiders hunting rate on $E$. flabellatus \\
$v$ & Spiders hunting rate on A. fuscicollis \\
$w$ & Spiders hunting rate on $P$. oleae \\
$L$ & Spiders carrying capacity \\
$z$ & Spiders net reproduction rate \\
$q_{i}$ & Spiders conversion by $i$ th prey capture, $i \in\{E, A, M\}$ \\
$a$ & E. flabellatus parasitism rate on $A$. fuscicollis \\
$b$ & E. flabellatus parasitism rate on $P$. oleae \\
$c$ & A. fuscicollis parasitism rate on $P$. oleae \\
$e$ & E. flabellatus conversion by A. fuscicollis parasitization \\
$h$ & E. flabellatus conversion by $P$. oleae parasitization \\
$r$ & E. flabellatus net reproduction rate \\
$K$ & E. flabellatus carrying capacity \\
$g$ & A. fuscicollis conversion by $P$. oleae parasitization \\
$f$ & A. fuscicollis intraspecific competition rate \\
$s$ & $P$. oleae net reproduction rate \\
$m_{A}$ & A. fuscicollis natural mortality rate \\
$k_{i}$ & Extra mortality rates due to pesticide effects, $i \in\{S, E, A, M\}$ \\
$U$ & P. oleae carrying capacity \\
\hline
\end{tabular}

Thus, in the absence of spraying, i.e. $k_{S}=k_{E}=k_{A}=k_{M}=0$, this equilibrium is always feasible.

Then $P_{3}=\left(S_{3}, E_{3}, 0,0\right)$, where

$S_{3}=\frac{L\left(K q_{E} r u-K k_{E} q_{E} u-k_{S} r+r z\right)}{K L q_{E} u^{2}+r z}$,

$E_{3}=\frac{K\left(L k_{S} u-L u z-k_{E} z+r z\right)}{K L q_{E} u^{2}+r z}$.

This equilibrium is feasible if and only if the following conditions hold

$\frac{z}{L u}\left(k_{E}-r\right)<k_{S}-z<-\frac{u q_{E} K}{r}\left(k_{E}-r\right)$.

Thus for $k_{E}>r$ the point is unfeasible, while in the absence of spraying, the feasibility condition reduces to

$L u<r$.

Next we find $P_{9}=\left(S_{9}, 0,0, M_{9}\right)$, with

$S_{9}=\frac{L\left(U q_{M} s w-U k_{M} q_{M} w-k_{S} s+s z\right)}{L U q_{M} w^{2}+s z}$,

$M_{9}=\frac{U\left(L k_{S} w-L w z-k_{M} z+s z\right)}{L U q_{M} w^{2}+s z}$

which is feasible for

$\frac{z}{L w}\left(k_{M}-s\right)<k_{S}-z<-\frac{U w q_{M}}{s}\left(k_{M}-s\right)$

and again for $k_{M}>s$ the equilibrium $P_{9}$ is unfeasible. When no insecticide is used, feasibility simplifies as follows

$L w<s$

For $P_{10}=\left(0, E_{10}, 0, M_{10}\right)$ we have

$E_{10}=\frac{K\left(U b h s-U b h k_{M}-k_{E} s+r s\right)}{K U b^{2} h+r s}$,

$M_{10}=\frac{U\left(K b k_{E}-K b r-k_{M} r+r s\right)}{K U b^{2} h+r s}$

admissible if and only if the following conditions are met

$\frac{r}{b K}\left(k_{M}-s\right)<k_{E}-r<-\frac{h b U}{s}\left(k_{M}-s\right)$

and once more for $k_{M}>s$ this point is unfeasible. For $k+M=k_{E}=0$, the feasibility condition becomes

$b K<s$.
Similar considerations hold for $P_{12}=\left(0,0, A_{12}, M_{12}\right)$, for which we find $A_{12}=\frac{U c g s-U c g k_{M}-k_{A} s-m_{A} s}{U c^{2} g+f s}$,

$M_{12}=\frac{U\left(c k_{A}+c m_{A}-f k_{M}+f s\right)}{U c^{2} g+f s}$.

The equilibrium point is feasible if and only if the following conditions are met

$\frac{s}{c}\left(k_{M}-s\right)<k_{A}+m_{A}<-\frac{g c U}{s}\left(k_{M}-s\right)$

from which infeasibility follows for $k_{M}>s$. If the insecticide is not used, feasibility is attained for

$m_{A}<g c U$.

For the points at which just one population vanishes, analytic expressions for the population values can be obtained, and are contained in Appendix A. These equilibria are $P_{11}=\left(S_{11}, E_{11}, 0, M_{11}\right), P_{13}=$ $\left(S_{13}, 0, A_{13}, M_{13}\right)$ and $P_{14}=\left(0, E_{14}, A_{14}, M_{14}\right)$. The coexistence equilibrium instead cannot be assessed and its analysis is obtained through numerical simulations. As seen, not all the possible combinations between the presence and the absence of a population arise. Ecologically, this can be explained by the fact that $A$. fuscicollis, being a specialist predator, cannot thrive in the absence of its prey $P$. oleae.

\subsubsection{Stability}

The Jacobian of (2.1) is

$J=\left[\begin{array}{cccc}J_{1,1} & u q_{E} S & v q_{A} S & w q_{M} S \\ -u E & J_{2,2} & e a E & h b E \\ -v A & -a A & J_{3,3} & g c A \\ -w M & -b M & -c M & J_{4,4}\end{array}\right]$

where:

$$
\begin{aligned}
J_{1,1}= & u q_{E} E+v q_{A} A+w q_{M} M \\
& +z-2 \frac{z}{L} S-k_{S}, \\
J_{2,2}= & e a A+h b M+r \\
& -2 \frac{r}{K} E-u S-k_{E}, \\
J_{3,3}= & g c M-m_{A}-2 f A \\
& -a E-v S-k_{A}, \\
J_{4,4}= & -b E-c A+s \\
& -2 \frac{s}{U} M-w S-k_{M} .
\end{aligned}
$$

Let us also introduce the following notation. The square matrix $J_{\left[i_{k} ; j_{k}\right]}$ of order $\ell$ is obtained by retaining the $i_{k}$-th and $i_{k}$-th rows and columns, $k=1, \ldots, \ell$.

Here we report the results for the easier equilibria, where the stability conditions are obtained explicitly and postpone to Appendix A the analysis of the most complicated ones. In any case, all possible equilibria are found to be conditionally stable. Note that the particular case of no insecticide spraying affects somewhat the stability. However, with the exception of the cases that are explicitly mentioned below, the modifications in the conditions is trivial, amounting to setting the extra killing rate to zero, but does not provide particular additional insights in them. Thus, these cases are not explicitly listed. Table 2 summarizes the equilibria behaviors.

Equilibrium $P_{0}$

The eigenvalues of the Jacobian at $P_{0}$ are $z-k_{S}, r-k_{E},-\left(m_{A}+k_{A}\right)$, $s-k_{M}$ giving the stability conditions:

$z<k_{S}, \quad r<k_{E}, \quad s<k_{M}$

Evidently, in the absence of spraying this equilibrium is unconditionally unstable.

Equilibrium $P_{1}$ 
At $P_{1}$ the eigenvalues of the Jacobian are $-z+k_{S},-u S_{1}+r-k_{E}$, $-v S_{1}-\left(m_{A}+k_{A}\right),-w S_{1}+s-k_{M}$. Using the feasibility conditions, stability is ensured by

$r<u S_{1}+k_{E}, \quad s<w S_{1}+k_{M}$.

Equilibrium $P_{2}$

At $P_{2}$ the Jacobian's eigenvalues are $u q_{E} E_{2}+z-k_{S},-r+k_{E}$, $-a E_{2}-\left(m_{A}+k_{A}\right),-b E_{2}+s-k_{M}$. The stability conditions using feasibility reduce to

$u q_{E} E_{2}+z<k_{S}, \quad s<b E_{2}+k_{M}$.

\section{Equilibrium $P_{3}$}

The Jacobian's explicit eigenvalues for $P_{3}$ are $-a E_{3}-m_{A}-k_{A}-v S_{3}$ and $-b E_{3}+s-w S_{3}-k_{M}$. From the latter the submatrix $J_{[1,2 ; 1,2]}$ of order two is left for which we find that both Routh-Hurwitz conditions hold:

$$
\begin{aligned}
\operatorname{tr}\left(J_{[1,2 ; 1,2]}\right) & =-\frac{z}{L} S_{3}-\frac{r}{K} E_{3}<0, \\
\operatorname{det}\left(J_{[1,2 ; 1,2]}\right) & =\frac{z}{L} \frac{r}{K} S_{3} E_{3}+u^{2} q_{E} S_{3} E_{3}>0 .
\end{aligned}
$$

The stability conditions are therefore $m_{A}+a E_{3}+k_{A}+v S_{3}>0$ which is trivially satisfied and

$s<b E_{3}+w S_{3}+k_{M}$

\section{Equilibrium $P_{8}$}

For $P_{8}$ the eigenvalues of the Jacobian are $w q_{M} M_{8}+z-k_{S}, h b M_{8}+$ $r-k_{E}, g c M_{8}-\left(m_{A}+k_{A}\right), k_{M}-s<0$, the latter inequality stemming from the feasibility condition (3.3). The stability conditions are thus

$$
\begin{array}{r}
w q_{M} M_{8}+z<k_{S}, \quad h b M_{8}+r<k_{E}, \\
g c M_{8}<m_{A}+k_{A} .
\end{array}
$$

Again, here we discover that no spraying entails the instability of this equilibrium. The same result is achieved if spiders are not affected by the insecticide.

Equilibrium $P_{9}$

For $P_{9}$ the explicit eigenvalues are: $h b M_{9}+r-k_{E}-u S_{9}$ and $g c M_{9}-$ $m_{A}-k_{A}-v S_{9}$. By deleting the second and third rows and columns of the Jacobian the Routh-Hurwitz conditions applied to $J_{[1,4 ; 1,4]}$ are both satisfied, reducing indeed to

$$
\begin{aligned}
\operatorname{tr}\left(J_{[1,4 ; 1,4]}\right) & =-\frac{z}{L} S_{9}-\frac{s}{U} M_{9}<0, \\
\operatorname{det}\left(J_{[1,4 ; 1,4]}\right) & =\left[\frac{z}{L} \frac{s}{U}+w^{2} q_{M}\right] M_{9} S_{9}>0 .
\end{aligned}
$$

The stability conditions reduce then just to

$$
\begin{aligned}
& h b M_{9}+r<k_{E}+u S_{9}, \\
& g c M_{9}<m_{A}+k_{A}+v S_{9} .
\end{aligned}
$$

\section{Equilibrium $P_{10}$}

For $P_{10}$ we find the explicit eigenvalues $w q_{M} M_{10}+u q_{E} E_{10}+z-k_{S}$ and $g c M_{10}-m_{A}-k_{A}-a E_{10}$, while for the remaining minor $J_{[2,4 ; 2,4]}$ the Routh-Hurwitz conditions once again hold unconditionally,

$$
\begin{aligned}
\operatorname{tr}\left(J_{[2,4 ; 2,4]}\right) & =-\frac{r}{K} E_{10}-\frac{s}{U} M_{10}<0, \\
\operatorname{det}\left(J_{[2,4 ; 2,4]}\right) & =\left[\frac{r}{K} \frac{s}{U}+b^{2} h\right] E_{10} M_{10}>0 .
\end{aligned}
$$

The stability conditions are therefore:

$$
\begin{aligned}
& w q_{M} M_{10}+u q_{E} E_{10}+z<k_{S}, \\
& g c M_{10}<m_{A}+k_{A}+a E_{10} .
\end{aligned}
$$

This point too, in view of the first condition (3.19), becomes unstable when insecticide is not used or even if the spraying does not harm the spiders.
Table 2

Summary of feasibility and stability conditions of the equilibrium points of the model.

\begin{tabular}{lll}
\hline Equilibrium & Feasibility & Stability \\
\hline$P_{0}=(0,0,0,0)$ & - & $z<k_{S}, r<k_{E}, s<k_{M}$ \\
$P_{1}=\left(S_{1}, 0,0,0\right)$ & $z>k_{S}$ & \\
$P_{2}=\left(0, E_{2}, 0,0\right)$ & $r>k_{E}$ & \\
$P_{3}=\left(S_{3}, E_{3}, 0,0\right)$ & $(3.4)$ & \\
$P_{8}=\left(0,0,0, M_{8}\right)$ & $s>k_{M}$ & \\
$P_{9}=\left(S_{9}, 0,0, M_{9}\right)$ & $(3.6)$ & (3.17) \\
$P_{10}=\left(0, E_{10}, 0, M_{10}\right)$ & $(3.8)$ & (3.19) \\
$P_{11}=\left(S_{11}, E_{11}, 0, M_{11}\right)$ & $($ A.1) \\
$P_{12}=\left(0,0, A_{12}, M_{12}\right)$ & $(3.10)$ & (A.6), (A.8), (A.9) \\
$P_{13}=\left(S_{13}, 0, A_{13}, M_{13}\right)$ & (A.2), (A.3) \\
$P_{14}=\left(0, E_{14}, A_{14}, M_{14}\right)$ & (A.4), (A.5) \\
$P_{15}=\left(S_{15}, E_{15}, A_{15}, M_{15}\right)$ & not analyzed & (A.15), (A.17) \\
\hline
\end{tabular}

Table 3

Available data on the other insects. The first and second row data are from Villa et al (2016b). Rows three to six: P. oleae data are from Villa et al. (2016a). Rows 5 and 6 data for E. flabellatus are from Villa et al. (2017a). Row 5 and 6 data for A. fuscicollis are from Villa et al. (2017b).

\begin{tabular}{llll}
\hline & P. oleae & E. flabellatus & A. fuscicollis \\
\hline $\begin{array}{l}\text { Probability of an insect } \\
\text { emerging from a pupa }\end{array}$ & $\mathbb{P}_{\mathbb{M}}=0.205$ & $\mathbb{P}_{\mathbb{E}}=0.152$ & $\mathbb{P}_{\mathbb{A}}=0.194$ \\
\hline $\begin{array}{l}\text { Insects emerging } \\
\text { from a pupa }\end{array}$ & 1 & 2.31 & 11.83 \\
\hline $\begin{array}{l}\text { Eggs laid } \\
\text { (optimal diet) }\end{array}$ & 195.79 & & \\
\hline $\begin{array}{l}\text { Eggs laid } \\
\text { (underfed diet) }\end{array}$ & 56.89 & & \\
\hline Longevity & $25.16 \approx 25$ & $49.61 \approx 50$ & $6.15 \approx 6$ \\
\hline Mortality & 0.04 & 0.02 & $m_{A}=0.166666666$ \\
\hline
\end{tabular}

\subsubsection{Verification and model behavior}

We address here the issue about the factual existence of the equilibria. As some of the stability conditions are quite involved, and in some cases the feasibility conditions too, it is legitimate to ask the question whether these conditions are indeed verified and do not define the empty set. Now, the equilibria with just one population are all easily attainable as both feasibility and stability conditions simultaneously hold for suitable parameter choices, that are not here reported. Those with two nonvanishing populations are also attained. We report for each one of them a parameter set that satisfies them. Note that for the parameters that can be assessed, we use the values that can be obtained from field data, reported below in Table 3, namely (3.28), (3.29), (3.30), (3.31), and always the following hypothetical initial conditions:

$S_{0}=58.500, \quad E_{0}=90.09695$,

$A_{0}=80.57839, \quad M_{0}=40.09695$.

For $P_{9}$, we have the set

$$
\begin{aligned}
q_{E} & =0.0073, \quad q_{A}=0.34, \quad q_{M}=4.5, \\
u & =0.1, \quad v=0.2, \quad w=0.0003, \\
h & =0.03, \quad k_{S}=0.000023, \quad a=1.002, \\
b & =0.0085, \quad c=0.000234, \\
r & =10^{-4}, \quad k_{E}=0.01, \quad f=1.00133, \\
K & =10, \quad k_{A}=10^{-6}, \quad k_{M}=10^{-7} .
\end{aligned}
$$

$P_{10}$ is obtained by the choice

$q_{E}=0.0073, \quad q_{A}=0.34, \quad q_{M}=1.5$, 

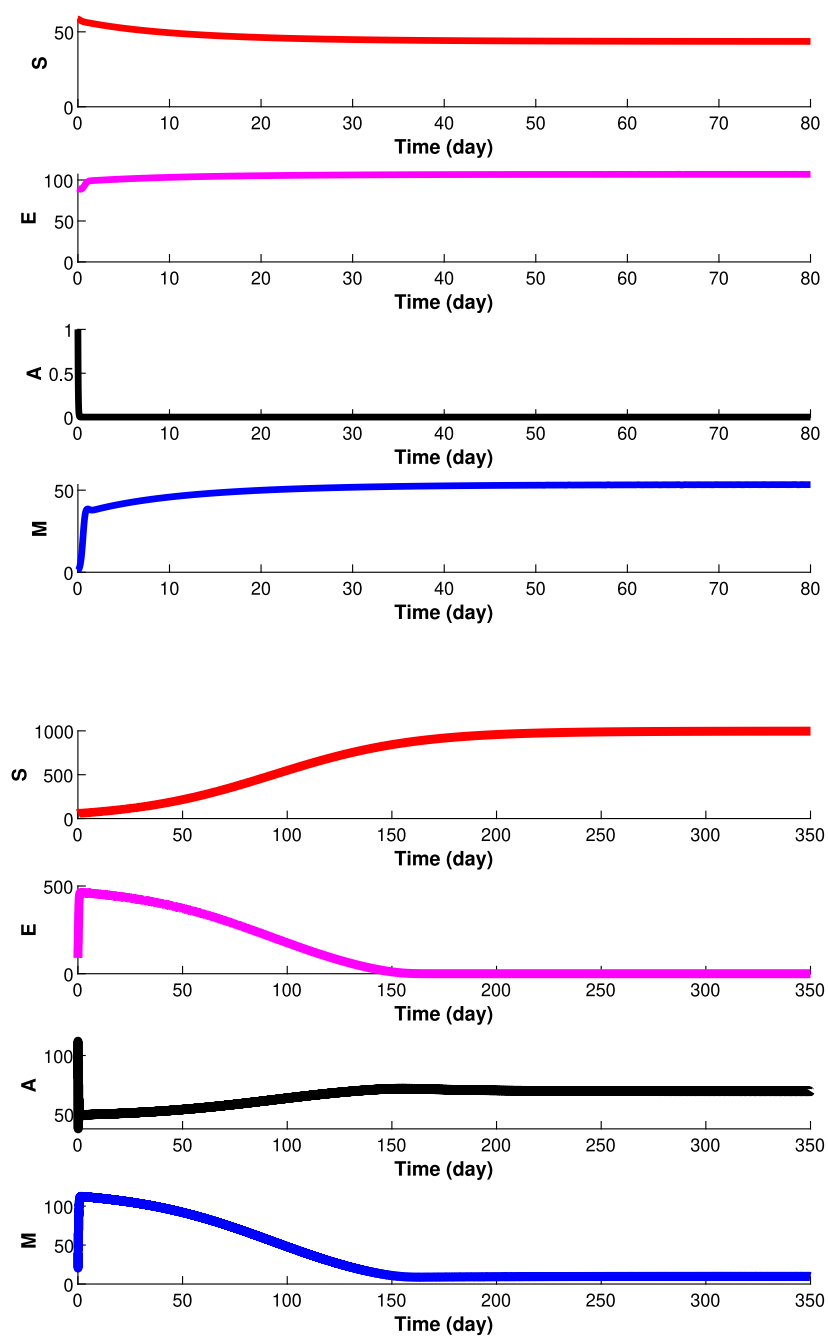

Fig. 1. We use (3.20), (3.28), (3.29), (3.30) and (3.31). Top to bottom the populations $S, E, A, M$ as function of time (measured in days). Upper frame: simulation for $P_{11}$, attained for the parameter values (3.25); Lower frame: simulation for $P_{13}$, attained for the parameter values (3.26).

$$
\begin{aligned}
u & =0.01, \quad v=0.2, \quad w=0.0003, \\
h & =0.03, \quad k_{S}=40, \quad a=1.05672, \\
b & =0.0085, \quad c=0.00234, \quad r=5.5, \\
k_{E} & =0.1, \quad f=2.00133, \\
K & =100, \quad k_{A}=k_{M}=10 .
\end{aligned}
$$

For $P_{12}$ the needed parameter values are

$$
\begin{aligned}
q_{E} & =0.0073, \quad q_{A}=8, \quad q_{M}=4.5, \\
u & =0.1, \quad v=0.2, \quad w=0.0003, \\
h & =0.03, \quad k_{S}=40, \quad a=5.05672, \\
b & =5.0085, \quad c=2, \quad r=4, \\
k_{E} & =40, \quad f=2.00133, \\
K & =100, \quad k_{A}=0.01, \quad k_{M}=0.001 .
\end{aligned}
$$

$P_{3}$ is obtained via the choice

$$
\begin{aligned}
q_{E} & =0.0073, \quad q_{A}=0.34, \quad q_{M}=0.004, \\
u & =0.1, \quad v=0.2, \quad w=0.3, \\
h & =0.03, \quad k_{S}=0.01, \quad a=0.05672,
\end{aligned}
$$
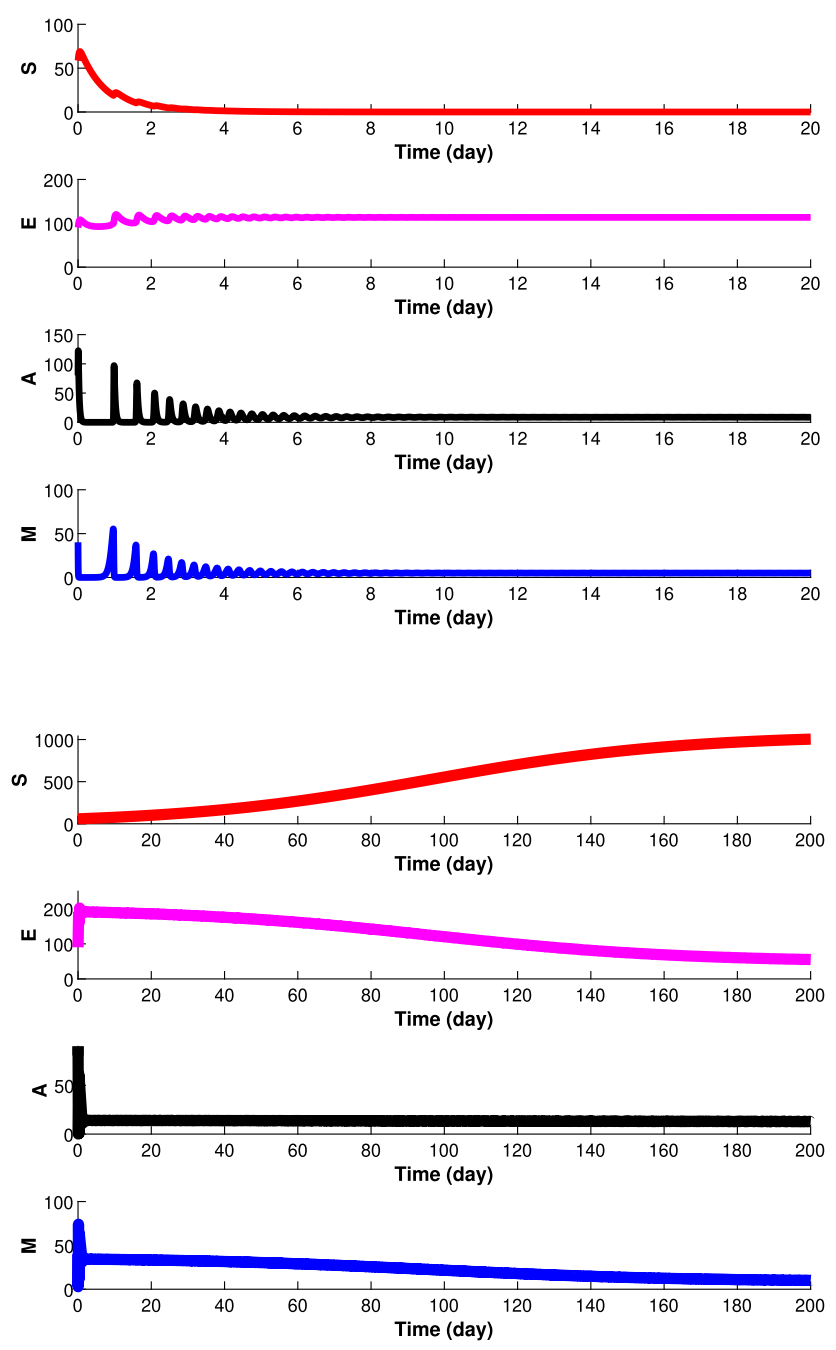

Fig. 2. We use (3.20), (3.28), (3.29), (3.30) and (3.31). Top to bottom the populations $S, E, A, M$ as function of time (measured in days). Upper frame: equilibrium $P_{14}$ is found for the set of parameters (3.26): note that the values of the populations at time $t=20$ are $S=0, E=113.4684, A=8.7195, M=5.0359$ and the last two keep away from zero also at later times. Indeed at time $t=200$, for instance, we find $A=8.6539, M=4.9770$. Lower frame: the coexistence equilibrium $P_{15}$ is stably obtained by choosing the parameters (3.27).

$$
\begin{aligned}
b & =2.0085, \quad c=1.00234, \\
r & =108, \quad k_{E}=10, \quad f=1.00133, \\
K & =50, \quad k_{A}=k_{M}=40 .
\end{aligned}
$$

The remaining equilibria are seen to arise for suitable hypothetical parameter choices, and the results are reported in Figs. 1-2.

Parameter values for $P_{11}$ :

$$
\begin{aligned}
q_{E} & =0.0073, \quad q_{A}=0.34, \quad q_{M}=0.004, \\
u & =0.01, \quad v=0.2, \quad w=0.3, \\
h & =0.08, \quad k_{S}=0.1, \quad a=0.1756, \\
b & =0.17565, \quad c=0.1756, \quad r=4.5 . \\
k_{E} & =0, \quad f=0.0001, \\
K & =100, \quad k_{A}=0.2, \quad k_{M}=0.5
\end{aligned}
$$

Parameter values for $P_{13}$ :

$$
\begin{aligned}
q_{E} & =0.00000073, \quad q_{A}=0.00073, \\
q_{M} & =0.0000073, \quad u=0.0073, \\
v & =0.01, \quad w=0.0073, \quad h=0.03,
\end{aligned}
$$



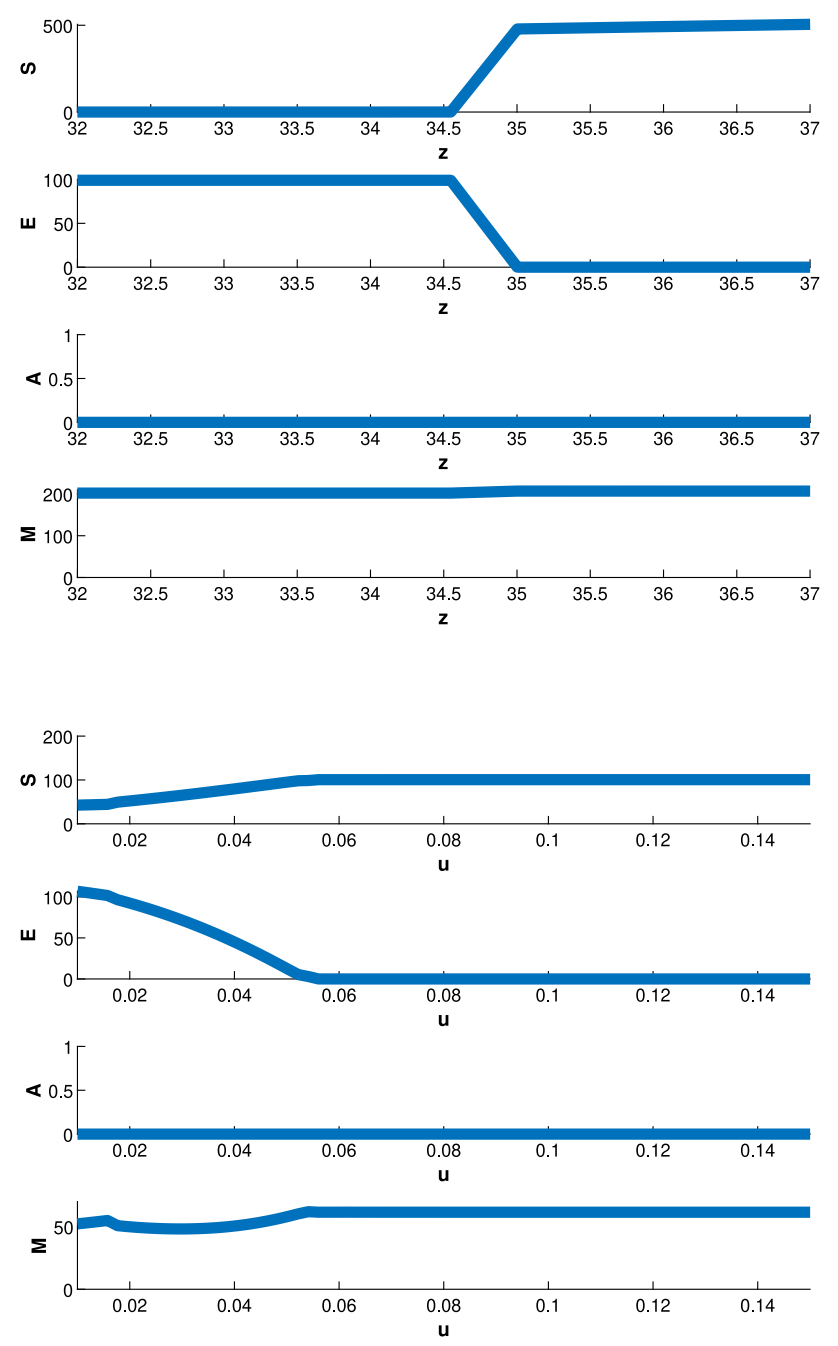

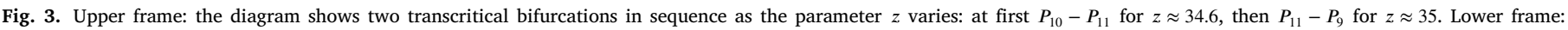
transcritical bifurcation $P_{11}-P_{9}$ as a function of $u$.

$$
\begin{aligned}
a & =0.25, \quad b=0.0022, \quad c=0.452, \\
r & =0.8, \quad k_{E}=1, \quad f=0.0001, \\
K & =100, \quad k_{S}=k_{A}=k_{M}=0
\end{aligned}
$$

Parameter values for $P_{14}$ :

$$
\begin{aligned}
q_{E} & =0.0073, \quad q_{A}=0.34, \quad q_{M}=0.004, \\
u & =0.01, \quad v=0.2, \quad w=0.3, \\
h & =0.08, \quad k_{S}=1.5, \quad a=0.1756, \\
b & =0.17565, \quad c=2.1756, \quad r=4.5, \\
k_{E} & =0, \quad f=0.1, \\
K & =100, \quad k_{A}=4, \quad k_{M}=0.5 .
\end{aligned}
$$

Parameter values for $P_{15}$ :

$$
\begin{aligned}
q_{E} & =q_{A}=q_{M}=0.0000073, \quad u=0.0068, \\
v & =0.00068, \quad w=0.0068, \quad K=100, \\
h & =0.35112, \quad k_{S}=0, \quad a=1.01, \\
b & =0.0022, \quad c=2.452, \quad r=5.012, \\
k_{E} & =k_{A}=k_{M}=0, \quad f=0.001 .
\end{aligned}
$$

\subsubsection{Bifurcations}

The model allows transitions from an equilibrium to another one, when suitable changes in the parameters occur. For instance, from Table 2, it is immediately seen that $P_{0}$ is incompatible with each one of the points $P_{1}, P_{2}$ and $P_{8}$, because its stability conditions are the opposite ones of the feasibility conditions for the latter set of points. This is an indication that transcritical bifurcations indeed occur. In this section we summarize the findings on this issue.

In fact, note that the model (2.1) shows several other transcritical bifurcations. They have been fully analyzed by means of Sotomayor's Theorem (Perko, 2011), and the details are deferred to Appendix B. In addition a few of them have been found by numerical simulations, and are reported in Figs. 3-5. It is interesting to note that from $P_{10}$, the Elasmus-moth equilibrium, the spiders can invade if their reproduction rate grows past the value 34.6, but if it grows further past 35, Elasmus is wiped out and only spiders and moth persist, Fig. 3 bottom.

In addition to transcritical bifurcations, also persistent oscillations can be determined, originated by suitable Hopf bifurcations. In particular, analytical conditions for the existence of such bifurcations are given in Appendix B, for the equilibria $P_{13}$ and $P_{14}$. For coexistence instead, the oscillatory behavior of the model has been discovered numerically. Indeed, the occurrence of a Hopf bifurcation at coexistence is shown in Fig. 5 right. 

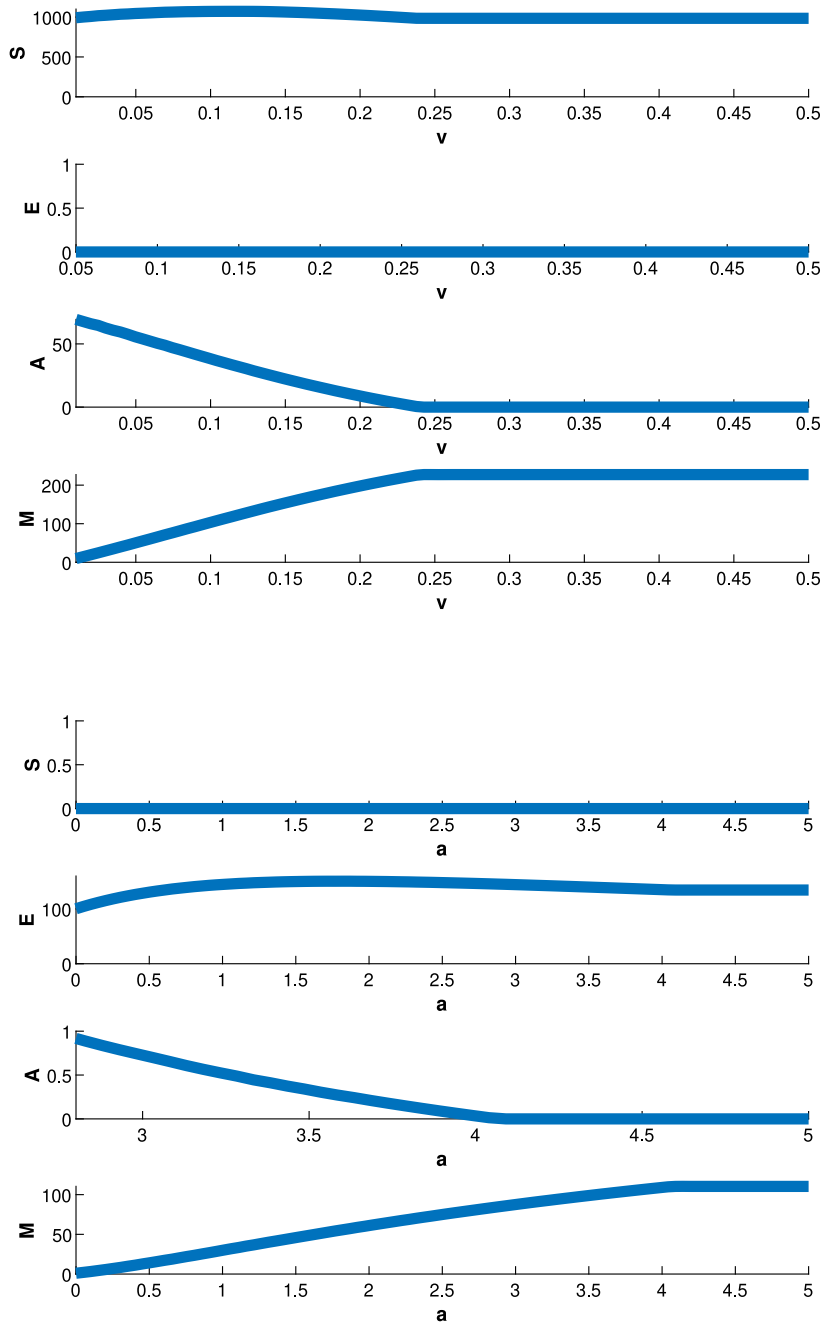

Fig. 4. Upper frame: transcritical bifurcation $P_{13}-P_{9}$ as a function of $v$ Lower frame: transcritical bifurcation $P_{14}-P_{10}$ as a function of $a$.

Finally, in Fig. 6 we provide a full picture illustrating the general relationships among the equilibria through transcritical bifurcations.

\subsection{Simulation of realistic scenarios}

We now turn to investigate the possible behavior of the system for the ecological application. The first step is the assessment of the model parameters from the available field data.

\subsubsection{Parameters assessment}

For the spider population, only their density at the beginning and at the end of the spring is known, respectively 58.500 and 476.500 individuals per hectare (Benhadi-Marín et al., 2020). By fitting on these data the simple logistic equation

$\frac{d S}{d t}=z S\left(1-\frac{S}{L}\right)$

we obtain the estimates for their reproduction rate and carrying capacity, that give $S(92)=476.4991$, which is acceptable. We thus take the values

$z=0.029368, \quad L=980$.

For the other insects, the available field data are reported in Table 3. These parameters include the probability that each insect species has of emerging from a pupa, the mean number of emerged insects from
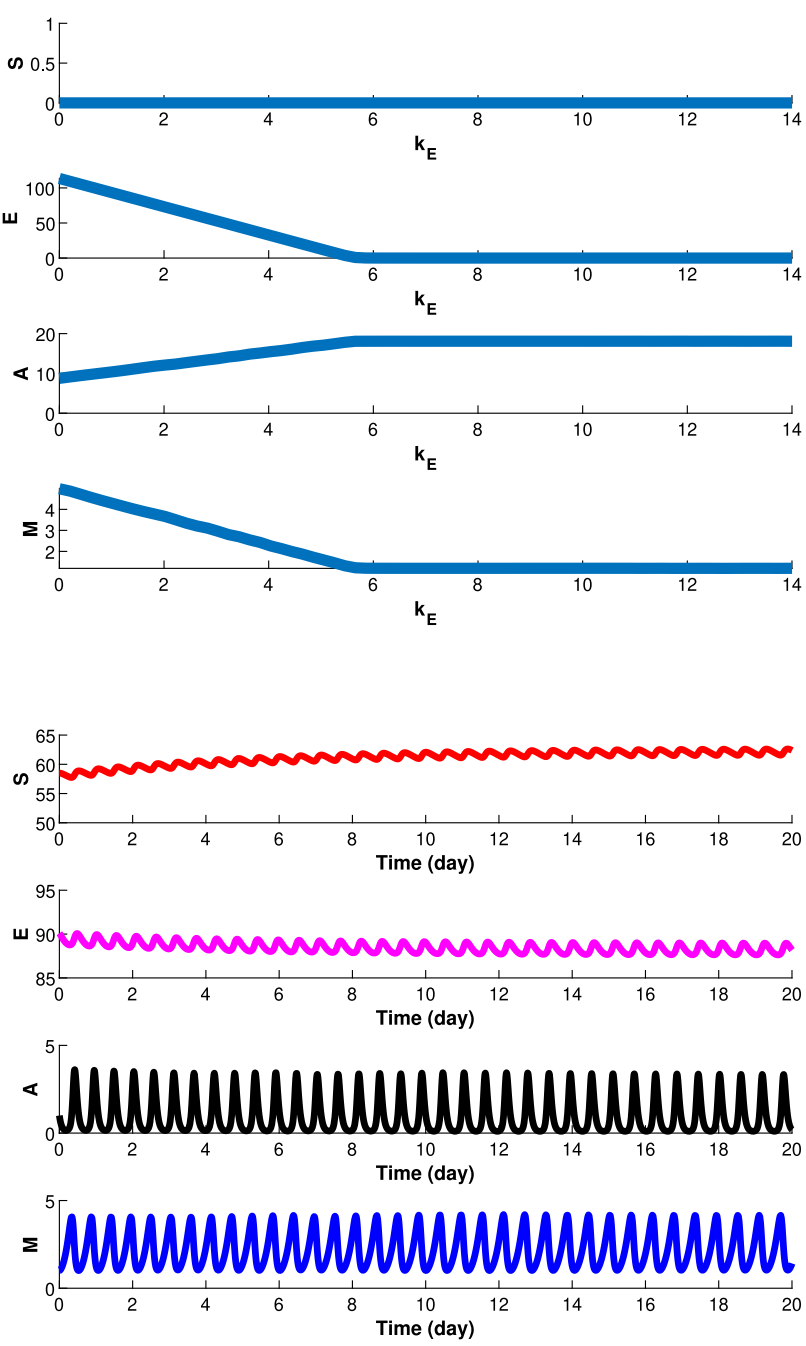

Fig. 5. Upper frame: Transcritical bifurcation $P_{14}-P_{12}$ as a function of $k_{E}$ Lower frame: Persistent oscillations around the coexistence equilibrium. It is obtained for the parameter values $q_{E}=0.0073, q_{A}=0.34, q_{M}=0.004, u=0.01, v=0.2, w=0.3, h=0.08$, $k_{S}=0.1, a=0.1756, b=0.17565, c=5.5, r=4.5, k_{E}=0, f=0.0001, K=100, k_{A}=0.2$, $k_{M}=0.5$ that almost entirely differ from those of (3.27).

a pupa, the number of eggs laid under an optimal and underfed diet, and the longevity and mortality rates. The probability with which each insect species emerges from a pupa and the number of insects emerging from a pupa were retrieved from previous experimental research conducted by Villa et al. (2016b) that collected pupae of $P$. oleae in olive orchards and kept them in controlled conditions until emergence. The probability of one of the species emerging from a pupa was estimated considering the emerged organism (i.e. $P$. oleae or the parasitoids, $E$. flabellatus or A. fuscicollis), the total number of pupae, and the nonemerging pupae. The number of insects emerging from a pupa was retrieved from the same work. In the case of $P$. oleae, one individual emerges from each pupa. In the case of the parasitoids, up to 2.31 and 11.83 individuals of E. flabellatus and A. fuscicollis can emerge on average, respectively. Longevity and mortality data were retrieved from Villa et al. (2016a) in the case of $P$. oleae, from Villa et al. (2017a) in the case of $E$. flabellatus, and from Villa et al. (2017b) in the case of $A$. fuscicollis. In these studies, several biological parameters were derived under laboratory conditions. In the case of $P$. oleae, the number of laid eggs was also recorded. Unfortunately, this parameter is not available for the parasitoids. Now, this information is used to obtain values for some other parameters as follows. 


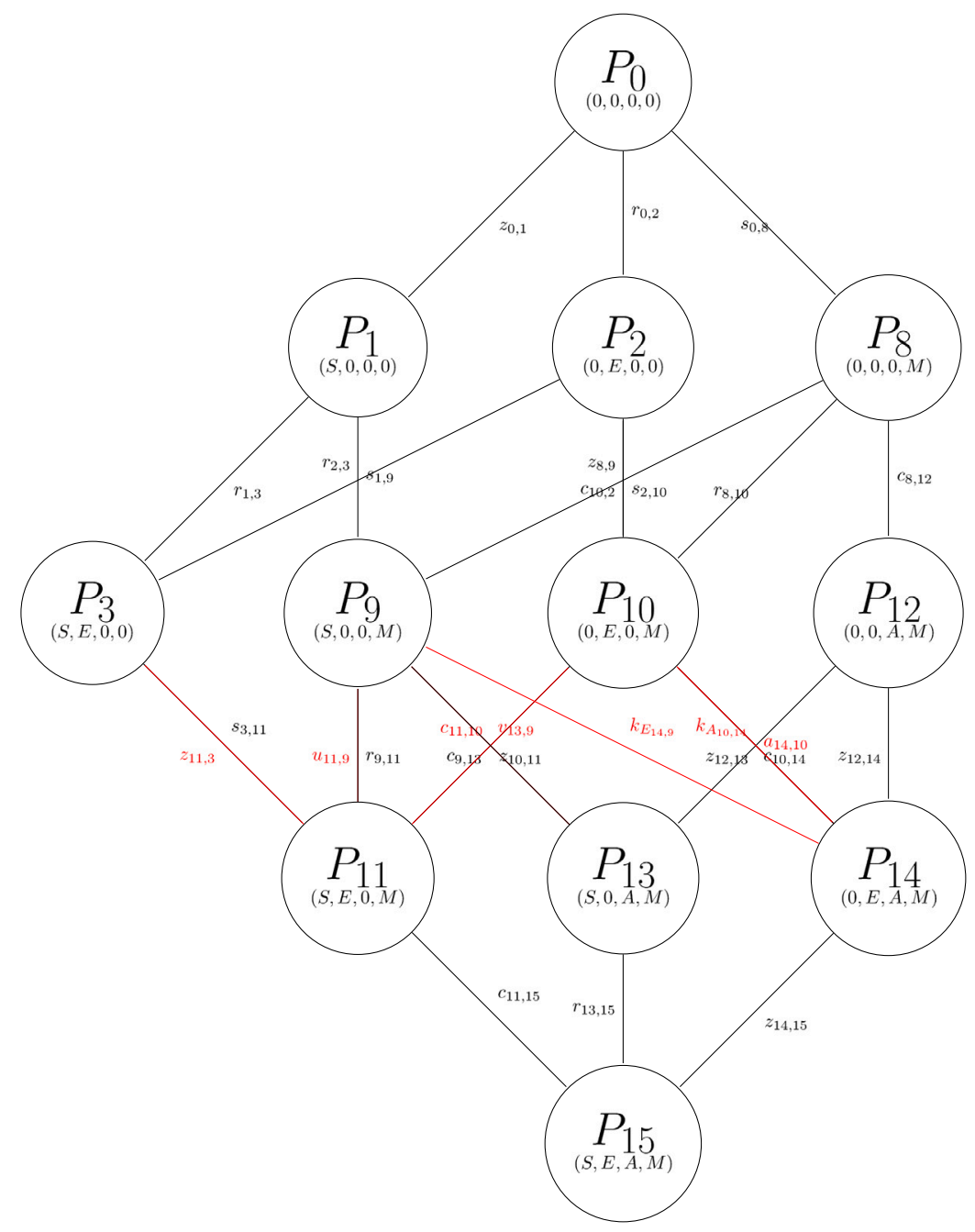

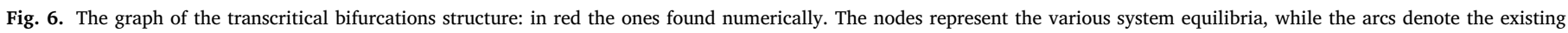

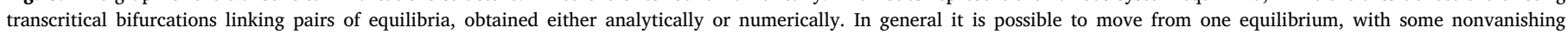

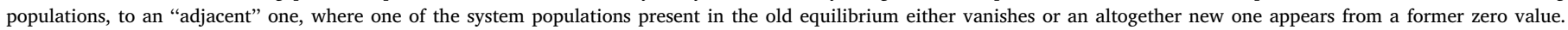

For the olive moth, we only consider the case of an optimal diet. The net birth rate $s$ is the difference between the birth rate and the mortality rate. The birth rate $\hat{s}$ is the product of the number of eggs laid by a moth in its lifetime with the probability that the emerging larvae become indeed adults, namely

$\hat{s}=195.79 \times 0.205=40.13695$,

$s=40.13695-0.04=40.09695$.

Elasmus reproduces by parasitizing $A$ and $M$ but the number of eggs laid is unknown, thus an estimate of the net reproduction rate $r$ is not possible. Also for Ageniaspis the number of eggs laid is not available. In a similar way we treat the corresponding coefficient $g$ for Ageniaspis, so that we have

$e=2.31 \times 0.152=0.35112$,

$g=11.83 \times 0.194=2.29502$.

Based on Bento (1999), we finally take the following value for the moths carrying capacity:

$U=277$,

and on previous records of olive moth eggs in the region.

\subsubsection{Possible climatic changes}

We discuss now how the ecosystem could be affected by possible climatic changes.
Table 4

Percentage of $P$. oleae egg mortality under various temperature and relative humidity conditions.

Source: Data taken from Bueno (1981).

\begin{tabular}{llllll}
\hline Temperature & \multicolumn{3}{c}{ Relative } & \multicolumn{3}{c}{ humidity } \\
\cline { 2 - 6 } & 50 & 60 & 70 & 80 & 90 \\
\hline 20 & 100 & 21.5 & 9.8 & 12 & 1.1 \\
25 & 100 & 64.3 & 7.3 & - & 1.2 \\
30 & 100 & 100 & 83.9 & 49.3 & 13.4 \\
35 & 100 & 100 & 100 & 94.9 & 93.1 \\
40 & 100 & 100 & 100 & 100 & 100 \\
\hline
\end{tabular}

To this end, we assume that the system parameters will change if the temperature raises. Specifically, for $P$. oleae we assume that the reproduction rate becomes lower with increasing humidity while instead it increases with raising temperature, at any given degree of humidity. This is based on the data reported in Table 4 (Bueno, 1981).

It is difficult to specifically quantify this information within the model, because the same change in reproduction could arise under different combinations of humidity and temperature. We therefore assume various degrees of $P$. oleae reproduction rate reduction, namely starting from $s$, we consider in sequence $s / 2, s / 4, s / 6, s / 8$ and $s / 10$. At the same time we assume that climatic-induced changes may not or may occur in the parameters of the other species. The results for the various 

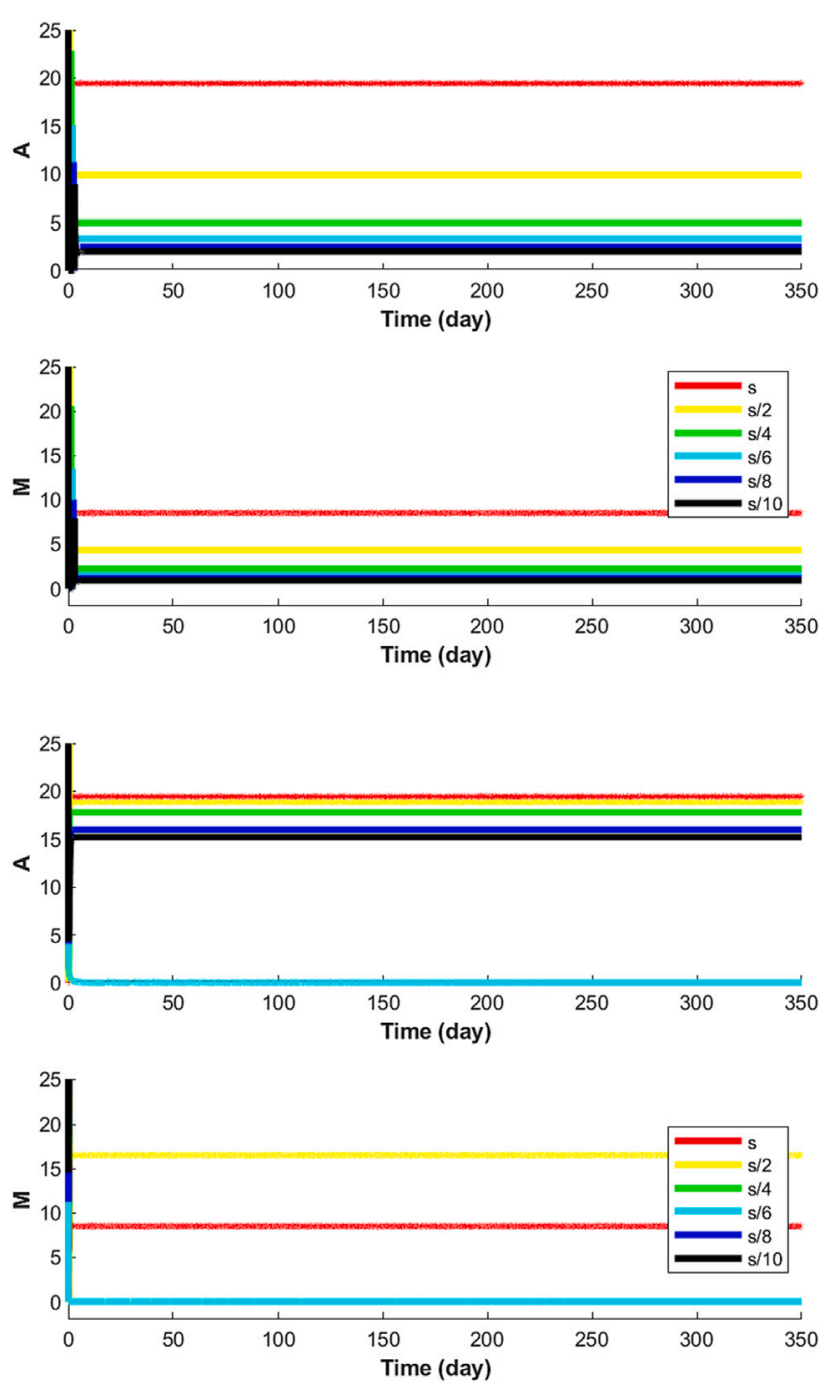

Fig. 7. Effects of climatic changes for the Ageniaspis-Prays-only equilibrium, $P_{12}$. Top A, bottom $M$. The red trajectories represent the current system behavior, with parameters given by (3.23), (3.20), (3.28), (3.29), (3.30), (3.31). The other colors indicated in the legend represent the system trajectories under climate changes that supposedly induce a reduction in one or all insects growth rates, as specified below. Upper frame: only the moth growth rate $s$ is affected; Lower frame: the growth rates of all species are affected in the same way as for moths.

equilibria are reported in Figs. 7-12, where on the top we always report the changes only for the moth. On the bottom instead all populations growth rates are assumed to change as the moths do. Namely, we change, in the same way as $s$ does, the reproduction parameters and the ones related to hunting/parasitizing as well, because reproduction is strictly related to the ability of prey/host capturing or parasitizing. Specifically, they are $u, v, w, z$ for $S, a, b, r$ for $E, c$ for $A$. With the exception of these parameters that case by case change, all these figures are obtained with the parameter values given in (3.21), (3.22), (3.23), (3.25), (3.26), (3.26), (3.27), and of course those that never vary, (3.20), (3.28), (3.29), (3.30), (3.31).

For the Ageniaspis-Prays-only equilibrium, $P_{12}$, in Fig. 7 we observe that the levels of both species steadily drop with diminishing moth reproduction rates. The moth population in this situation gets reduced tenfold in the worst situation while Ageniaspis are essentially eradicated, in case only the moth growth rate is affected. When instead all insects suffer the climatic changes, Ageniaspis behaves in the same way, but its reduction is only a third of the standard value, while the moth drops up to being almost eradicated. However, it is interesting to note
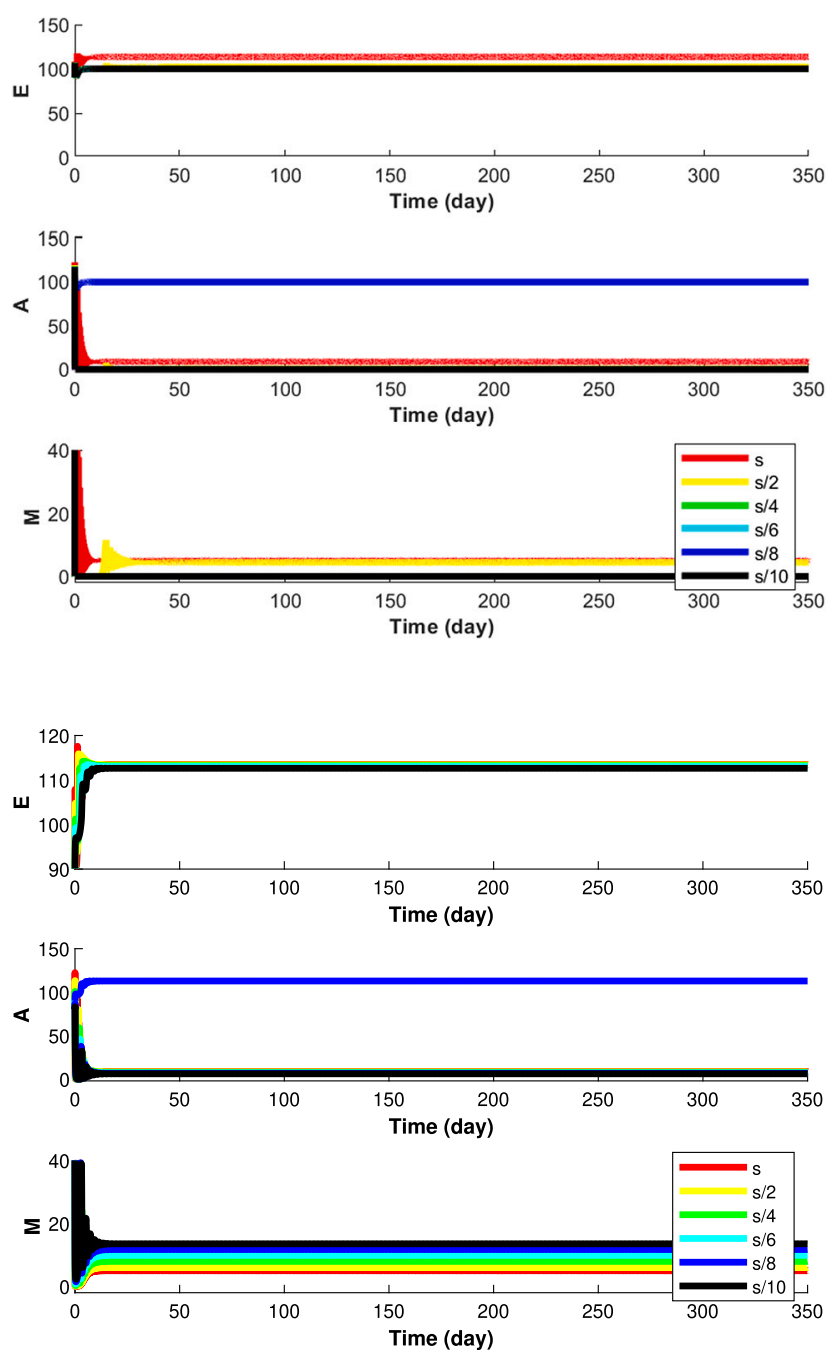

Fig. 8. Effects of climatic changes for the spider-free equilibrium $P_{14}$. Top $E$, center $A$, bottom $M$. Note that in this column the vertical scale for Elasmus starts from 90 and not from 0 , The red trajectories represent the current system behavior, with parameters given by (3.26), (3.20), (3.28), (3.29), (3.30), (3.31). The other colors indicated in the legend represent the system trajectories under climate changes that supposedly induce a reduction in one or all insects growth rates, as specified below. Upper frame: only the moth growth rate $s$ is affected; Lower frame: the growth rates of all species are affected in the same way as for moths. Note that in this column the vertical scale for Elasmus starts from 90 and not from 0, to better show the differences in the graphs.

that the behavior is more complex, because for a half of the standard parameter values, Ageniaspis is slightly reduced, while the Prays population raises of about $50 \%$. For a fifth of the standard parameter values, both populations essentially disappear. Finally when the parameters attain a tenth of their standard reference value, Ageniaspis rebounds to a third of the starting value, as said above, while Prays attains values slightly different from zero.

For the spider-free equilibrium $P_{14}$, Fig. 8 top, Elasmus drops steadily up to about $13 \%$ of the standard reference value, with decreasing moth reproduction rates, as does the moth itself, finally attaining vanishing values. For the Ageniaspis instead at first we observe a decrease, but then there is a rather large rebound for $s / 8$, raising the population about 5 times from the standard reference level and then another drop for $s / 10$ to almost vanishing values. When all insects growth rates are affected by climatic changes, Fig. 8 bottom, Elasmus is scantly affected, Ageniaspis shows a similar behavior that it has when only $s$ changes, but the rebound for $s / 8$ attains only the population level corresponding to the original value of $s$. For Prays instead a steady increase is observed 

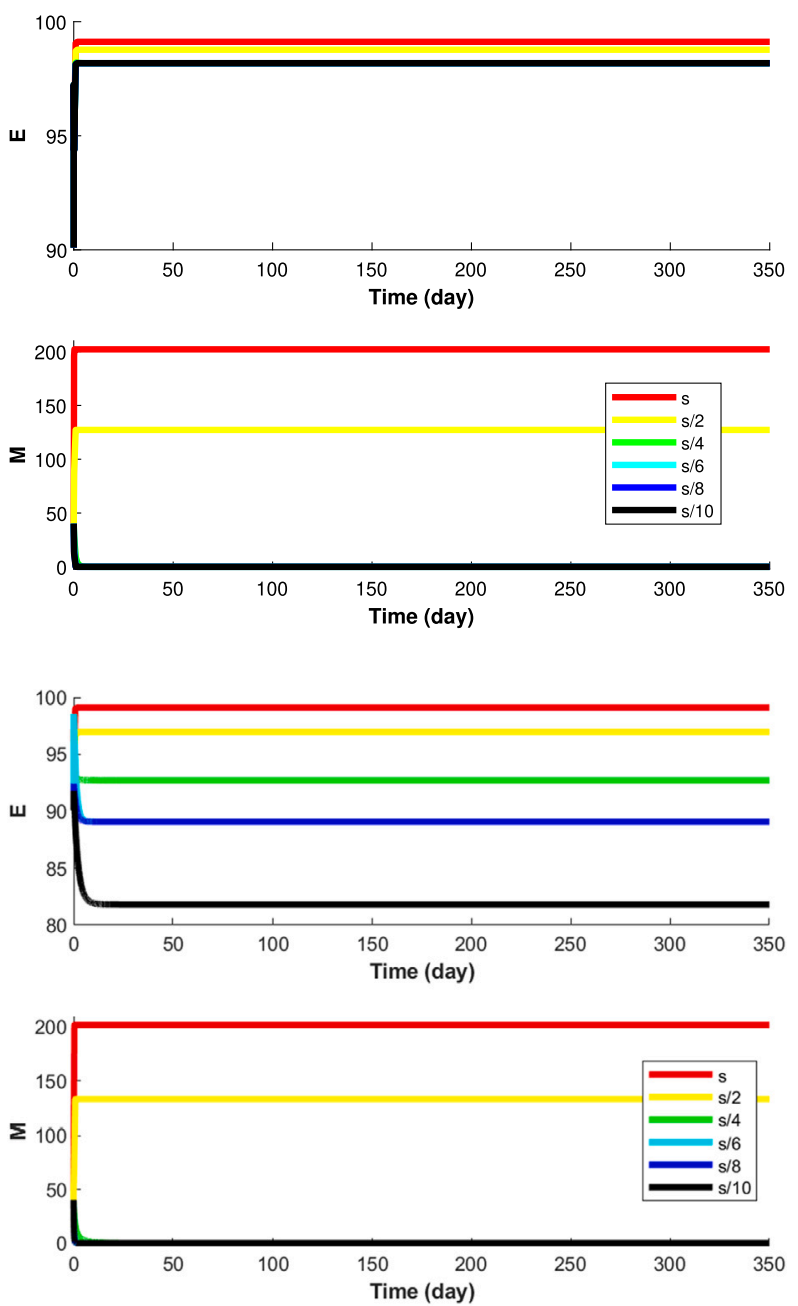

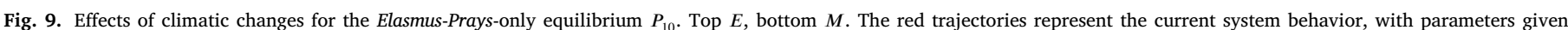

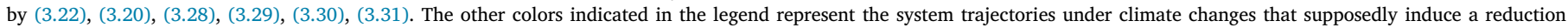

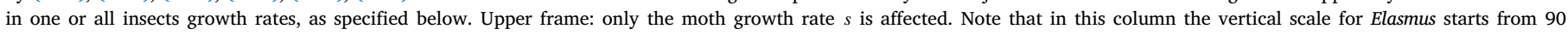

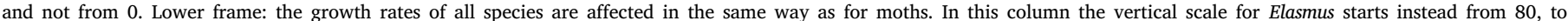
better show the differences in the graphs.

with a decrease in the parameters, leading to a final value that doubles the standard reference level.

For the Elasmus-Prays-only equilibrium $P_{10}$, Fig. 9, when only the moth growth rate is affected, Elasmus drops about 3\% while Prays instead initially drops about a fourth of the standard reference for $s / 2$, and then for further reductions of this parameter, it is essentially eradicated. A similar behavior is observed also in case when all insects suffer climatic influence, but Elasmus drops instead about $17 \%$.

In case of the Elasmus-free point $P_{13}$, Fig. 10, spiders are not affected by the moth growth rate changes, Ageniaspis and moths, after a steady decrease, are essentially eradicated when the maximum reduction in the parameter $s$ occurs. When all insects growth rates are affected by temperature and humidity changes, with the reduction of the reproduction parameters, the spiders population seems to rise slower, at least initially, Ageniaspis are scantly affected, the moth population at equilibrium instead experiences a steady increase.

For the Ageniaspis-free point $P_{11}$, Fig. 11, by substantial changes only in the moth growth rate, spiders and moths are essentially wiped out, while Elasmus gets reduced about $10 \%$. When all insects feel the effect of climatic variability, spiders again after a steady decrease disappear, Elasmus instead increases steadily about $25 \%$, and also Prays rebound gradually to double their equilibrium values,
The spider-moth-only point shows a steady decrease of both populations with decreasing $s$, the former by about $50 \%$ the latter by $44 \%$, Fig. 12. If all insects are subject to climate influence, both populations equilibrium values do not change, only the speed at which these values are attained do appear to be affected, being slowed down by the lower parameter values.

For coexistence, by changes only in the moth growth rate, spiders are not affected, Elasmus, Ageniaspis and moths are reduced and finally essentially wiped out. When all insects feel the effect of climatic variability, spiders are significantly reduced, although an increasing trend is observed in the figure, perhaps meaning that only the speed at which equilibrium is reached is slowed down. The remaining populations again show a decreasing trend that pushes them to very low values, Fig. 13.

\section{Discussion}

The olive tree damage caused by the olive moth is associated with the feeding behavior of its different generations. The anthophagous generation destroys a variable amount of flowers during the olive tree blooming, reducing the amount of set fruits. The carpophagous generation is responsible for the fruit drop in two periods, the first after the fruit setting in June/July and the second one starting at the 

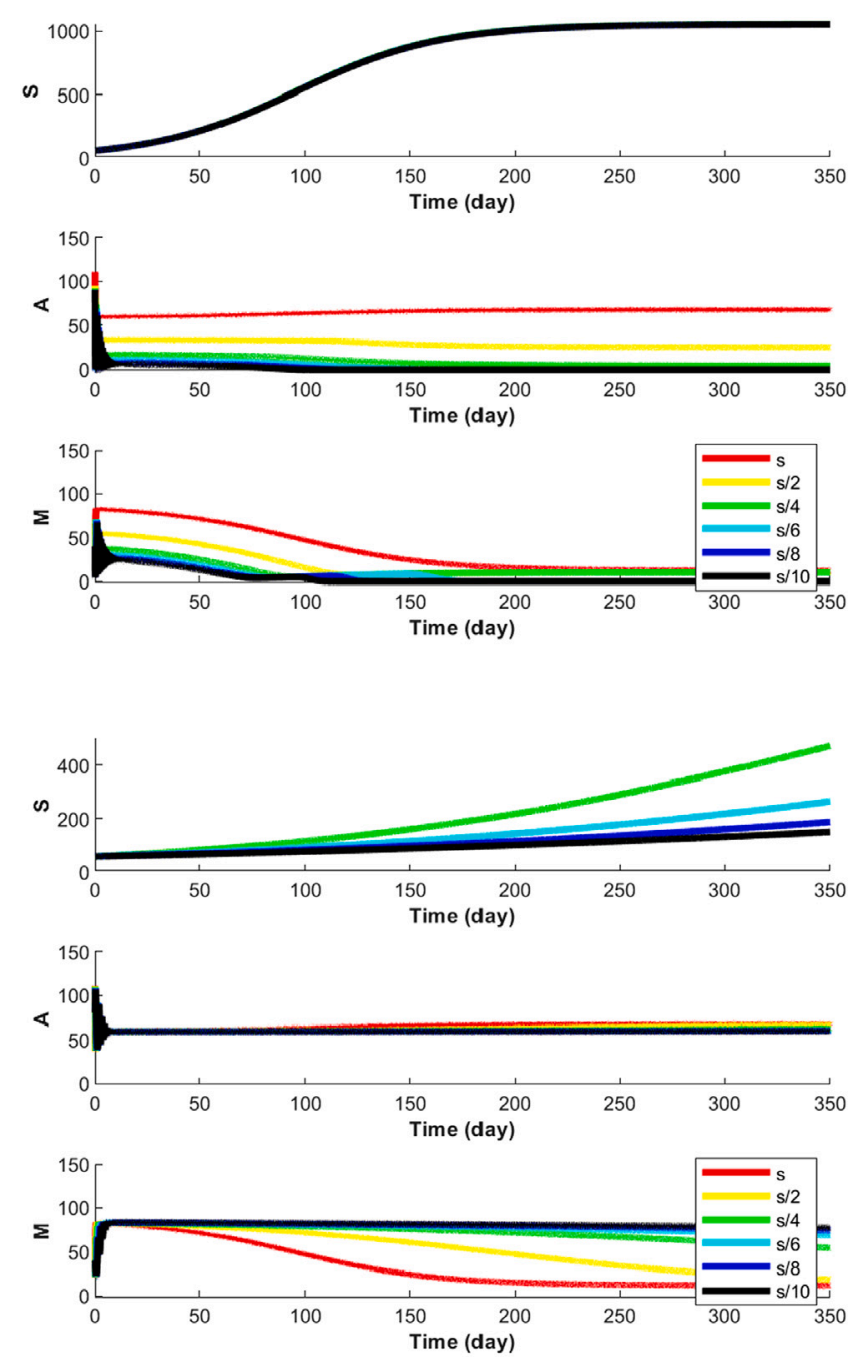

Fig. 10. Effects of climatic changes for the Elasmus-free equilibrium $P_{13}$. Top $S$, center $A$, bottom $M$. The red trajectories represent the current system behavior, with parameters given by (3.26), (3.20), (3.28), (3.29), (3.30), (3.31). The other colors indicated in the legend represent the system trajectories under climate changes that supposedly induce a reduction in one or all insects growth rates, as specified below. Upper frame: only the moth growth rate $s$ is affected; Lower frame: the growth rates of all species are affected in the same way as for moths.

beginning of September. The phylophagous generation usually does not originate important fruit losses, although severe attacks may negatively affect the tree development (Bento, 1999).

In view of these remarks, we now investigate the system equilibria to draw inferences on how to fight this olive pest.

The system can attain any one of the 12 equilibria listed in Table 2. But in what follows we should remember that the points $P_{0}, P_{8}, P_{12}$ and $P_{14}$ are achievable only with a substantial use of insecticides. We discuss at first the ones where the olive moth disappears.

\subsection{The pest-free cases}

At $P_{0}=(0,0,0,0)$ we observe the extinction of the four insect species, including $P$. oleae. Thus, the olive grove is free from the pest. This equilibrium is always admissible and turns out to be stable if and only if each extra mortality rate $k_{i}$ with $i=S, E, A, M$, caused by the pesticides, is larger than the net reproduction rate of the corresponding population.

From the biological point of view, this equilibrium is achieved if the olive groves are subjected to curative treatments based on insecticides.
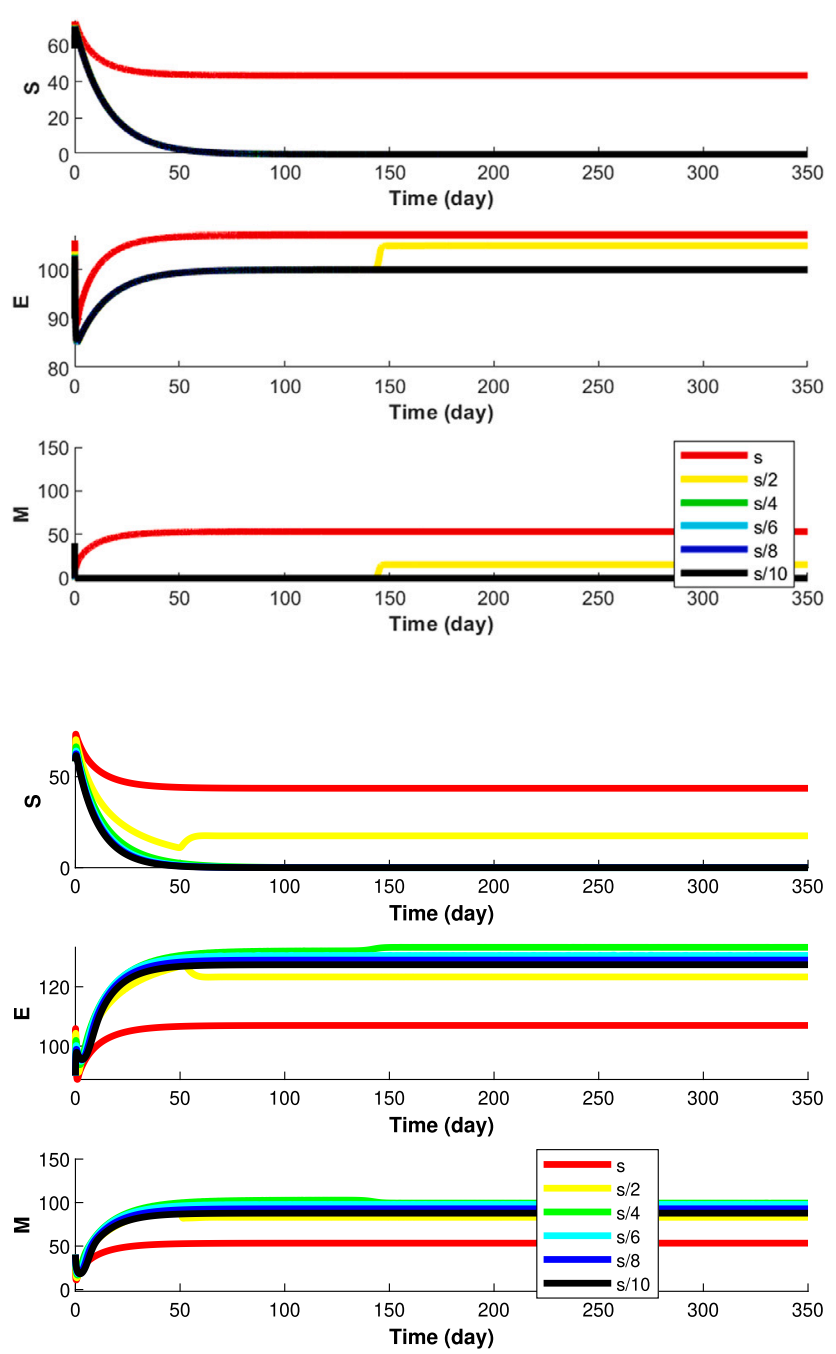

Fig. 11. Effects of climatic changes for the Ageniaspis-free equilibrium $P_{11}$. Top $S$, center $E$, bottom $M$. The red trajectories represent the current system behavior, with parameters given by (3.25), (3.20), (3.28), (3.29), (3.30), (3.31). The other colors indicated in the legend represent the system trajectories under climate changes that supposedly induce a reduction in one or all insects growth rates, as specified below. Upper frame: only the moth growth rate $s$ is affected; Lower frame: the growth rates of all species are affected in the same way as for moths.

The authorized pesticides in Portugal for the olive moth control are based on the neonicotinoid acetamiprid; the pyrethroids cypermethrin, deltamethrin and lambda-cyhalothrin; the organophosphate phosmet; and spinetoram, a multi-component tetracyclic macrolide in the class of spinosyn insecticides (DGAV, 2021). However, pesticides may be responsible for serious toxic effects on human health and the environment. They remain in the ecosystem and hamper the sensitive environmental equilibrium through bio-accumulation, reaching nontarget organisms such as humans and pests' natural enemies (Sharma et al., 2020). For example, Pitzer et al. (2021) describes acute and chronic effect of deltamethrin on human brain and behavior. Alternatively, the use of sexual pheromones were investigated but the results indicate that they would be useful and effective only with low populations levels. On the contrary, Bacillus thuringiensis can be used under organic production systems against the anthophagous generation. It was indeed found to reduce the pest up to 80 to $90 \%$,

Equilibrium $P_{1}=\left(S_{1}, 0,0,0\right)$ is better than the former, because the spiders survive. Spiders are generalist predators that feed mainly on insects. They are important natural enemies of relevant pests in 

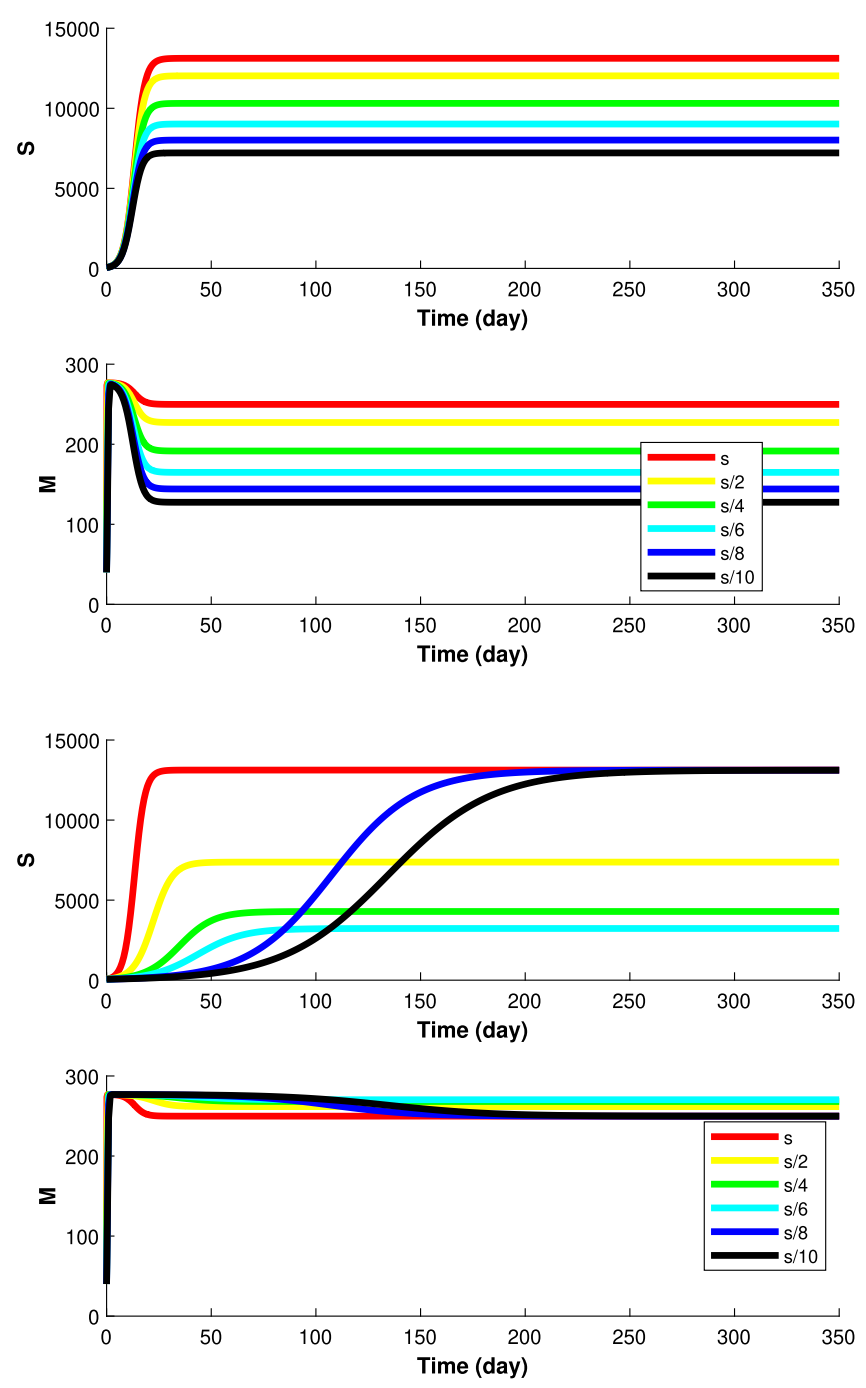

Fig. 12. Effects of climatic changes for the Elasmus-Ageniaspis-free equilibrium. Top $S$, bottom $M$. The red trajectories represent the current system behavior, with parameters given by (3.21), (3.20), (3.28), (3.29), (3.30), (3.31). The other colors indicated in the legend represent the system trajectories under climate changes that supposedly induce a reduction in one or all insects growth rates, as specified below. Upper frame: only the moth growth rate $s$ is affected; Lower frame: the growth rates of all species are affected in the same way as for moths.

agroecosystems because of their ubiquity and abundance (BenhadiMarín et al., 2016). Usual agricultural management often relies on the use of pesticides, tillage, fertilization or landscape simplification. However, these practices may alter the diversity of spiders and their effectiveness as pest control agents (Benhadi-Marín et al., 2016). At first, for the equilibrium $P_{1}$ to be feasible, the average reproduction rate must exceed the extra mortality rate due to pesticides $z>k_{S}$. Stability of $P_{1}$ is achieved by satisfying the conditions (3.14), which explicitly can be rewritten as $0<z\left(k_{E}+u L-r\right)<u L k_{S}$ and $0<z\left(k_{M}+w L-s\right)<$ $w L k_{S}$. Thus, the reproduction rates of the populations $E$ and $M$ must respectively be lower than the extra corresponding mortality rate due to the pesticide plus a certain positive amount that depends on the spider hunting rate, $r<k_{E}+u L, s<k_{M}+w L$. Further, the mortalities in these right hand sides must be bounded above by the same net reproduction augmented by an extra factor, namely $k_{E}+u L<u L k_{S} z^{-1}+r, k_{M}+w L<$ $w L k_{S} z^{-1}+s$.

At $P_{2}=\left(0, E_{2}, 0,0\right)$ only the E. flabellatus thrives thanks to the alternative nutritional resources in the environment. As for $P_{1}$, here the equilibrium is feasible if the net reproduction rate is larger than
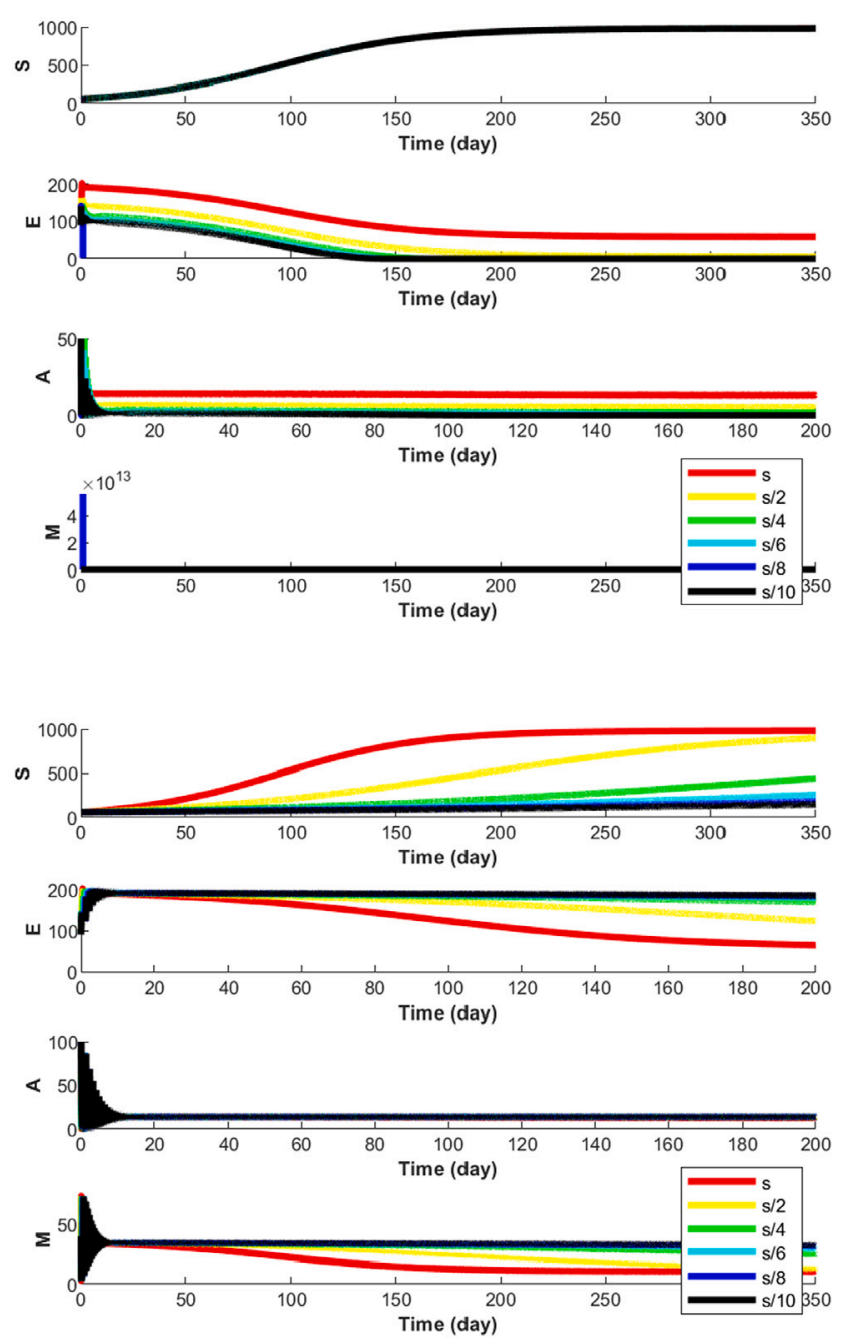

Fig. 13. Effects of climatic changes for the coexistence equilibrium. The red trajectories represent the current system behavior, with parameters given by (3.27), (3.20), (3.28), (3.29), (3.30), (3.31). The other colors indicated in the legend represent the system trajectories under climate changes that supposedly induce a reduction in one or all insects growth rates, as specified below. Upper frame: only the moth growth rate $s$ is affected; Lower frame: the growth rates of all species are affected in the same way as for moths.

the extra pesticide-induced mortality rate $r>k_{E}$. The increase in the number of $E$. flabellatus leads to an increased parasitism of the olive moth larvae. This entails a further decrease of the moth's adult population, leading to a reduction in fruit damage. Equilibrium $P_{2}$ is stable if and only if $0<r\left(z+u q_{E} K-k_{S}\right)<u q_{E} K k_{E}$ and $0<$ $r\left(k_{M}+b K-s\right)<b K k_{E}$. The second stability condition requires in particular that the moths net reproduction rate must be lower than the combined mortality rates due to pesticide use and E. flabellatus parasitism, the latter indicating the importance for moth eradication of this parasitoid. Generally, the effect of E. flabellatus on the olive moth has been understood as unwanted because the highest parasitism rates were found in the phyllophagous generation (attacking 10 to $11 \%$ of the olive moth) and because its hyperparasitic behavior which may reduce also the populations of $A$. fuscicollis. Villa et al. (2016a), found that in olive orchards with herbicide application where the emergence of A. fuscicollis was significantly lower than in olive orchards with spontaneous vegetation, E. flabellatus was responsible for almost half of the parasitism. This supports our results and highlights the nonnegligible effect of non-specific natural control agents (which can rely on other resources for survival), which have a higher impact on the 
particular situation of unfavorable conditions for the specific ones (such as low number of the pest or low food resources for adults).

At $P_{3}=\left(S_{3}, E_{3}, 0,0\right)$ the spiders and $E$. flabellatus populations coexist and biologically the situation for the olive trees is excellent with a population of parasitoids and predators persisting ready to fight possible pest infiltration. This equilibrium point undergoes a transcritical bifurcation with $P_{1}, P_{2}$ and $P_{11}$ when respectively the parameters $r, s$ and $z$ vary as reported in Fig. 6 . This equilibrium is unfeasible again if the pesticide extra mortality rate is larger than the E. flabellatus net reproduction rate. The free-pest scenarios highlights the ecological importance of generalist natural control agents such as the spiders or $E$. flabellatus. They may prove to be effective in the case of pest invasions of free-pest territories such as the Americas or Australia (CABI, 2021), where the olive growing is in expansion, or in the hypothetical case that the pest would become extinct in a territory where it is already present. However, in regions where the olive moth is well established the free-pest scenarios would be very unlikely. Therefore the scenarios described in the next subsection would apply.

\subsection{The moth persistence scenarios}

We now turn to the situations in which the olive pest is endemic. We try to discuss the conditions for which these equilibria would not be attainable, but one should keep in mind that if such an equilibrium becomes unfeasible or unstable, in general it is not possible to establish a priori where the system would settle moving away from it; thus it is very well possible that if one such pest-affected point is prevented to arise, the system would stabilize at another point where the pest is still present, so that eradication is not attained. The bifurcation diagram of Fig. 6 however could constitute a useful guide to the applied ecologist in the choice of the policy to be undertaken case by case.

\subsection{1. $P_{8}=\left(0,0,0, M_{8}\right)$}

At the equilibrium $P_{8}$ only the population of the $P$. oleae thrives. This is possible because it has essentially unlimited food resources within the olive agroecosytem. For its feasibility the insect net reproduction rate must exceed the extra mortality rate due to the pesticide, i.e. $s>k_{M}$. This is trivially satisfied in the absence of insecticide spraying. It is well known that in the absence of natural control agents, the size of the pest population grows rapidly starting in the spring until at the end of the season it reaches a value higher than 300 in real scenarios (Villa et al., 2021). For this equilibrium to be unachievable, we need to ensure that the feasibility or the stability conditions (3.17) must not hold. The former is implied by a spraying rate larger than the moth reproduction rate. Instead, instability is ensured by either one of the conditions $z+w q_{M} M_{8} \geq k_{S}, r+h b M_{8} \geq k_{E}$ or $g c M_{8} \geq m_{A}+k_{A}$. Thus $P_{8}$ cannot be attained if, in the third case, the $A$. fuscicollis feeding rate on moth is larger than its combined natural and pesticide-induced mortalities. The first case amounts to state that the combined spiders' growth rate due to their alternative resources and predation on the moth exceeds their death rate due to insecticides; the second possibility is the corresponding situation for E. flabellatus. Note that in the absence of spraying, both these conditions are satisfied. This result is somewhat counterintuitive, implying that the moth-only point is not naturally achievable. Thus the pest must certainly coexist with one of its natural enemies.

Note that from this point the only moth-free equilibrium achievable is the origin, by suitably acting on the moth reproduction rate $s$, compare Fig. 6. Modifications of either $z, r$ or $c$, i.e. the reproduction rates of spiders, A. fuscicollis and its parasitizing rate would move the system from $P_{8}$ to other equilibria where however the moth still thrives.

\subsection{2. $P_{9}=\left(S_{9}, 0,0, M_{9}\right)$}

Coexistence of spiders and $P$. oleae populations occurs at $P_{9}$. A sufficient condition for it to be unfeasible is given by a pesticideinduced extra moth mortality rate larger than its net reproduction rate. In the opposite case, for $s-k_{M}$ being positive, the inequalities in (3.6) could still be violated, by using the corresponding difference for the spiders. The occurrence of equilibrium $P_{9}$ can be prevented also by destabilizing it, violating the inequalities in (3.18). Thus the total $E$. flabellatus reproduction rate due to moth parasitism and alternative resources must exceed their total mortality due to spiders predation and pesticide action. Alternatively, a corresponding situation for $A$. fuscicollis should be ensured, so that its reproduction rate exceeds the natural as well as the combined mortality rates due to pesticides and spiders hunting. It is interesting to note that these conditions involve both species that do not appear in the equilibrium. Although somewhat counterintuitive, they can be explained by the fact that either one of these two species must invade this point, and this can occur only if the model parameters allow their thriving.

Direct moth eradication from this point can only be obtained by action on the parameter $s$, its reproduction rate, to attain $P_{1}$, the spideronly equilibrium, see Fig. 6 . Changes in $c, r, u$ and $v$ would all keep the moth in the ecosystem, although other populations would appear; changing $z$ has the same effect, but with the removal of spiders. Fig. 3 top, shows the bifurcation between $P_{11}$ and $P_{9}$ as $z$ increases, while in the bottom frame the same transition occurs in terms of $u$. Fig. 4 in the top frame depicts instead the transcritical bifurcation between $P_{9}$ and $P_{13}$ when the parameter $v$ changes.

\subsection{3. $P_{10}=\left(0, E_{10}, 0, M_{10}\right)$}

Violating the feasibility conditions for $P_{10}$, the point where $E$. flabellatus and moth thrive, can be obtained exactly as in the corresponding discussion for equilibrium $P_{9}$, by substituting the difference between the $E$. flabellatus reproduction rate and the spraying-induced mortality in place of the spiders' one. More easily, also the equilibrium is unfeasible again if the pesticide extra mortality rate is larger than the moth net reproduction rate. Also the discussion for instability parallels the one of the previous equilibrium. The spiders combined reproduction rates based on feeding on moth and E. flabellatus as well as alternative resources must exceed their spraying-induced mortality, or else the $A$. fuscicollis natural mortality and the ones due to insecticide action and E. flabellatus predation must be lower than their hunting rate on moths.

Moth eradication can be ensured only by reducing its reproduction rate $s$, and the system would move to the point $P_{2}$, where only $E$. flabellatus thrives, see Fig. 6. Acting on $a, z$ and $c$ would maintain the moth presence, together with some other species. A reduction of $r$ would lead to the extinction of $E$. flabellatus, but the moths would still persist. In Fig. 3 the bifurcations involving $P_{10}$ have already been described above when considering $P_{9}$. In addition, the bottom frame of Fig. 4 contains the transition from this point to $P_{14}$ when $a$ decreases.

\subsection{4. $P_{11}=\left(S_{11}, E_{11}, 0, M_{11}\right)$}

For the A. fuscicollis-free point $P_{11}$ the interpretation of both feasibility and stability conditions is rather hard. However, the equilibrium is certainly unstable if condition (A.9) is violated. This can be achieved by requiring that the $A$. fuscicollis growth rate by moth feeding exceeds its combined natural plus spray-induced plus spiders' and E. flabellatus predation related mortalities. In such situation, however, it is likely that $A$. fuscicollis invades the system and thus coexistence is attained. Therefore pest eradication would not be achieved. Hence, despite the presence of two natural control agents, the pest thrives, this being probably due to the fact that the two natural control agents have alternative nutrients on which they can feed. From Fig. 6 it is seen that three more transcritical bifurcations may occur at this point, on of which in particular leads to the moth-free point $P_{3}$. Therefore, destabilizing $P_{11}$ by violating one of the remaining stability conditions (A.6) or (A.8) will eventually lead to the pest eradication. In view of their rather involved 
nature, however, it is hard to assess which condition to use, and also on which parameters to act to modify it.

From this point the moth can be eradicated by acting on $z$ or $s$, reducing the moth birth rate or increasing the spiders' one, thereby bringing the system to equilibrium $P_{3}$. Note that a large reduction of $z$ instead induces the disappearance of the spiders; the system would then attain $P_{10}$. Modifying instead $u$ and $r$ has the effect of removing one of the useful moth natural enemies, keeping the pest in the system. Acting on $c$ would still keep the moth alive, but depending on the change, either spiders disappear, at $P_{10}$ or $A$. fuscicollis invades the system and coexistence $P_{15}$ is attained, Fig. 6 .

From Fig. 6 it is evident that no change in any parameter would lead to direct moth eradication. Fig. 3 shows the two bifurcations involving $P_{11}$, taking the system to $P_{10}$ as $a$ decreases, and to $P_{9}$ when $u$ increases. already discussed been described above when considering $P_{9}$.

\subsection{5. $P_{12}=\left(0,0, A_{12}, M_{12}\right)$}

To guarantee unfeasibility of the spiders and $E$. flabellatus-free point $P_{12}$, if insecticide is used, the moth spraying-induced mortality being larger than their reproduction rate is sufficient. Further, suitable multiples of the difference between the moth reproduction rate and the corresponding insecticide killing rate establish a range, outside of which the sum of the natural and insecticide-caused mortalities of $A$. fuscicollis should lie in order to ensure unfeasibility. To violate stability of this equilibrium, the insecticide killing action should be smaller than the combined insects reproduction rates, coming by hunting their prey parasitizing their hosts or feeding on alternative resources, the insects being either spiders or E. flabellatus. In the absence of spraying, this equilibrium is unconditionally unstable, and therefore not achievable. It is likely that acting on $z$ the spiders birth rate due to other resources introduces in the system natural control agent populations, the spiders themselves or E. flabellatus, without however eradicating the pest. From Fig. 6 it is evident that no change in any parameter would lead to direct moth eradication.

\subsection{6. $P_{13}=\left(S_{13}, 0, A_{13}, M_{13}\right)$}

Feasibility of $P_{13}$ cannot be easily interpreted in ecological terms. In this case, to have instability, the spiders' conversion rate on moths should exceed the product of their conversion rate on A. fuscicollis and conversion rate of the latter on moths, but this is not sufficient. A sure way of unsettling this equilibrium is by ensuring that the total E. flabellatus growth rate by using alternative resources as well as by feeding on moths and $A$. fuscicollis is larger than their combined mortality due to spiders predation and insecticide action. In this second situation the likely outcome of increasing $r$ is the coexistence equilibrium, with the consequent non resolution of the pest problem, Fig. 6 . In this case too, moth eradication cannot be obtained without first moving toward other "neighboring" equilibria, see Figs. 5 and 6.

\subsection{7. $P_{14}=\left(0, E_{14}, A_{14}, M_{14}\right)$}

The feasibility of $P_{14}$ is again hard to interpret. Instability can be ensured by a low enough spider's killing rate due to the use of insecticides, which should be overcome by their combined reproduction rates on all their available resources, whether explicitly modeled or being alternative food supplies. In addition, one could exploit the situation in which the E. flabellatus conversion rate by feeding on moths could be larger than the product of their conversion rate on A. fuscicollis and conversion rate of the latter on moths, although by itself this condition does not guarantee instability.

From Fig. 6 it is not possible to directly remove the pest. Reducing $z$ as stated above has the effect of removing the E. flabellatus, thereby attaining the point $P_{12}$, while increasing it brings the system to coexistence. Further transitions appear in Figs. 4 and 5, respectively with $P_{10}$ and $P_{12}$.

\subsection{Final remarks}

The olive moth is subjected to the attack by several natural control agents in the olive agroecosystem. In the model presented here three of the most important organisms naturally feeding on the olive moth are represented. This model intends to elucidate how the abundance of $P$. oleae would change depending on corresponding variations in the population sizes of its natural enemies present in the ecosystem and accounted for in the equations. In the model a specific parasitoid, a generalist parasitoid and a generalist predator are included with the aim of gaining information about their effectivity for reducing the pest numbers. Their populations behaviors under different climate change scenarios are also considered. Thus, we compared the population levels attained in the various system equilibria. Starting from the coexistence, when one of the top two generalist natural enemies is removed and $A$. fuscicollis thrives, we observe that the pest population slightly changes (equilibria $P_{13}$ and $P_{14}$ ), although these results are highly affected by the effects of climate change. If instead $A$. fuscicollis is removed (equilibrium $P_{11}$, Fig. 11) the pest population suddenly explodes, reaching values about ten times higher than those attained at coexistence. The same phenomenon is observed when only two populations thrive, one of them being the olive moth. If the pest coexists with just $A$. fuscicollis ( $P_{12}$, Fig. 7), their equilibrium level is scantly affected. However, if they thrive either only with spiders or only with $E$. flabellatus (respectively $P_{9}$, Fig. $12 ; P_{10}$, Fig. 9), they are heavily affected and their number is highly increased.

In all cases the action of the climate change can be observed. Thus, at $P_{12}$, (the moth and A. fuscicollis both thriving) (Fig. 7) and $P_{14}$ (the spider-free point) (Fig. 8) the moths maintain always low levels. At $P_{10}$ (the E. flabellatus-moth equilibrium) (Fig. 9) a reduction of the moth to almost vanishing values is observed when the moth reproduction rate reduces between four- and tenfolds. These results indicate the contribution to the pest reduction of both parasitoids under a climate change scenario. Accordingly, at the E. flabellatus-free equilibrium $P_{13}$ (Fig. 10), the moths increase from almost extremely low values near 0 (with the normal moth reproduction rate) to 100 (with a tenfold reduction of its reproduction rate) and at the A. fuscicollis-free equilibrium $P_{11}$ (Fig. 11) the moths increase from 50 (with a normal moth reproduction rate) to 100 (after a 10 fold reduction of the moth reproduction rate), suggesting the relevance of the combined action of both parasitoids in the regulation process of the pest in the climate change context.

From these findings, it is apparent that the role of a specialist parasitoid in keeping the pest numbers in check is more important than the one of generalist predators, even if they act jointly in combination in a non-climate change scenario, but both seem to be important when the action of the climate change takes place. Additionally, the $P_{2}$ equilibrium indicates that under of unfavorable conditions for $A$. fuscicollis, such as very low number of the pest - which in certain years with unusual climatic conditions occurs, or low food resources for adults originated for example after the herbicide application (Villa et al., 2016b), or even invasions of $P$. oleae to free-pest territories (and therefore A. fuscicollis-free), the role of E. flabellatus (or other facultative parasitoids) could encompass a higher importance. This is in the line of Dainese et al. (2017) results, who found complementary effects among different guilds of natural enemies and an improvement of the biological control efficacy when increasing the non-crop areas (which may involve extra resources not explicitly considered in our model).

The equilibria $P_{0}$ - where all arthropods are absent, $P_{8}$ - where only the moth is present, $P_{12}$ - where A. fuscicollis and the moth are present, and $P_{14}$ - where $E$. flabellatus, $A$. fuscicollis and the moth are present, are not achievable without spraying. Additionally, in general the equilibria are not feasible if the pesticide extra mortality rate is larger than the net reproduction rate for all or some of the organisms involved. However, the available information about how pesticides affect to the mortality rates of the studied organisms is very limited or absent, pointing at 
the urgency of evaluating the effect of pesticides in natural control agents. The same occurs in the case of the effects of climate change. Here, we assumed the natural enemies would show a similar behavior to the olive moth, or that they would not be affected by climate change. However, a most probable real scenario would attain different results. For example, Villa et al. (2017a) indicate that A. fuscicollis hazard mortality rate highly increase with small variations of temperature. Therefore, both pesticides and climate change effects on the olive moth trophic web should be urgently investigated.

\section{Model limitations}

We present now a brief discussion of the possible criticism the proposed model could be subject to.

First of all, in the modeling process, using Occam's razor, we attempted to obtain a balance between the actual situation and a mathematically feasible compromise. Indeed, we completely disregarded the fact that insects undergo several development stages. Namely, we ignored the larval and pupa stages and we focused just on the adult individuals. This entails also that possible delays due for instance to hatching of eggs have been disregarded. Also, when developing dynamical models, we opted for having coefficients, i.e. model parameters, that are independent of time, i.e. in particular independent of the season. Using constant values for these coefficients means essentially that we focus just one season with suitable thriving conditions. This makes the system autonomous and renders the analysis possible. In other situations time-dependent parameters can be considered, see e.g. Rossini et al. (2021).

Furthermore, from the mathematical viewpoint, (2.1) suffers from the fact that the mutual pairwise interactions of the species are modeled as bilinear terms. This entails that there is no upper limit in how much a predator can eat of a captured prey. In reality, feeding is subject to a satiation phenomenon, for which, even in the presence of an unlimited amount of food, the actual intake rate drops and the amount eaten attains an upper value. This behavior is captured by a concave response function, called Holling type II (HTII), represented by a hyperbola which raises up from the origin to approach a horizontal asymptote. To introduce such expressions into (2.1) would make the model closer to reality, at the expense of complicating very much the mathematics, so that most likely the analysis would be altogether impossible, and only simulations would provide some information. Moreover, in the HTII formulation, the speed at which the plateau is approached depends on the attack rate. Indeed note that for the hyperbola $w(u)=c u(1+u)^{-1}$ the derivative is $w^{\prime}(u)=c(1+u)^{-2}$ and its evaluation at the origin $u=0$ gives $w^{\prime}(0)=c$. Thus $c$ here represents the attack rate, and the higher it is, the higher the slope and the faster the asymptote is approached. Moreover, the attack rate in turn largely relies on the encounter rate. The encounter rate in the field may not be large enough to imply a fast approaching of the asymptote. The hyperbola and its linear approximation in this case are therefore about the same. For these reasons, we opted for the current less complicated formulation (2.1).

The simulation of these models requires a deep knowledge about the behavior of the involved organisms in the field. In particular, the specific values of at least some of the parameters appearing in the model would be required. As it is apparent from Section 3.2, there is still an important gap in the information available so far. More dramatic is the lack of knowledge regarding the behavior of these organisms under a range of climate variables values changes, see Section 3.2.2. Additionally, not only the organisms itself, but also their different life cycle stages or different generations along the year may respond differently to changes in climate variables, similarly to the suggested by Pollard et al. (2020) for Phratora vulgatissima L. Therefore, increasing the laboratory and field research on this pest and its associated trophic web becomes an urgent matter. Furthermore, this model did not consider the effect that sex ratios may have on the organism populations, see e.g. Rossini et al. (2021). Finally, in field conditions the system is certainly much more complex. Here, we consider two important parasitoids and spiders as generalist predators. But it is known that other parasitoids or predators [e.g., the Chrysopideae Chrysoperla carnea (Stephens) or the Anthocoridae Anthocoris nemoralis (Fabricius)] may or do have important roles in the trophic web of the olive moth (Bento et al., 2007; Paredes et al., 2014).

\section{Conclusion}

The goal of this work is mainly to study the interaction between the natural enemies in the biological control of pest-infested olive trees, assessing the ecosystem steady states for feasibility and stability of a tritrophic system composed by the olive tree (the crop), the olive moth (the pest), A. fuscicollis (the specialist parasitoid), E. flabellatus (the facultative parasitoid) and spiders (generalist predators).

Thus we tried to analyze in detail, from a mathematical point of view, the possibilities to eradicate the pest to avoid possible damage to the olive tree crop.

Our results indicate that in normal circumstances the natural enemy which will perform the highest pressure over the olive moth is the specific parasitoid A. fuscicollis, and under particular conditions, $E$. flabellatus or spiders might have significant roles in controlling the pest.

In sum, our results indicate the high importance of specific parasitoids. The role of hyperparasitoids or generalist predators under particular circumstances must not be overlooked or disregarded. Additionally, we pointed out the huge gap of biological knowledge that still exists on the trophic web of the olive moth.

\section{Declaration of competing interest}

The authors declare that they have no known competing financial interests or personal relationships that could have appeared to influence the work reported in this paper.

\section{Acknowledgments}

This work has been partially supported by the projects "Metodi numerici in teoria delle popolazioni" and "Metodi numerici nelle scienze applicate" of the Dipartimento di Matematica "Giuseppe Peano" of the Università di Torino and by EU COST FA 1405 "Using three-way interactions between plants, microbes and arthropods to enhance crop protection and production". The authors are grateful to the Foundation for Science and Technology (FCT, Portugal) for financial support by national funds FCT to CIMO (UIDB/00690/2020). National funding by FCT, through the individual postdoc grant (SFRH/BPD/119487/2016) (María Villa).

\section{Appendix A. Equilibria assessment}

\section{A.1. Feasibility}

For $P_{11}=\left(S_{11}, E_{11}, 0, M_{11}\right)$ the population values can still be explicitly assessed. Letting

$$
\begin{aligned}
\Delta_{11}= & K L U b h q_{E} u w-K L U b q_{M} u w \\
& +K L q_{E} s u^{2}+K U b^{2} h z+L U q_{M} r w^{2}+r s z
\end{aligned}
$$

where $\Delta_{11}$ is the denominator of $E, M$ and $S$ and we put it $\Delta_{11}>0$, with $q_{E}>q_{M}$.

$$
\begin{aligned}
E_{11}= & \frac{N_{E_{11}}}{\Delta_{11}}, \quad M_{11}=\frac{N_{M_{11}}}{\Delta_{11}}, \quad S_{11}=\frac{N_{S_{11}}}{\Delta_{11}}, \\
N_{E_{11}}= & {\left[L U \left(b h k_{S} w-b h w z\right.\right.} \\
& +k_{M} q_{M} u w-k_{E} q_{M} w^{2}+q_{M} r w^{2}
\end{aligned}
$$




$$
\begin{aligned}
& \left.-q_{M} s u w\right)-U b h k_{M} z+U b h s z \\
& \left.+L k_{S} s u-L s u z-k_{E} s z+r s z\right] K, \\
N_{M_{11}}= & U\left(K L k_{E} q_{E} u w-K L k_{M} q_{E} u^{2}\right. \\
& -K L q_{E} r u w+K L q_{E} s u^{2}-K L b k_{S} u \\
& +K L b u z+K b k_{E} z-K b r z \\
& \left.+L k_{S} r w-L r w z-k_{M} r z+r s z\right), \\
N_{S_{11}}= & L\left[K U \left(b h q_{E} s u-b h k_{M} q_{E} u\right.\right. \\
& \left.-b^{2} h k_{S}+b^{2} h z+b k_{E} q_{M} w-b q_{M} r w\right) \\
& -K k_{E} q_{E} s u+K q_{E} r s u-U k_{M} q_{M} r w \\
& \left.+U q_{M} r s w-k_{S} r s+r s z\right] .
\end{aligned}
$$

The equilibrium point is $P_{11}$ is feasible if and only if

$$
\begin{array}{r}
N_{S_{11}} \Delta_{11}>0, \quad N_{E_{11}} \Delta_{11}>0, \\
N_{M_{11}} \Delta_{11}>0 .
\end{array}
$$

For $P_{13}=\left(S_{13}, 0, A_{13}, M_{13}\right)$ we find, defining

$$
\begin{aligned}
\Delta_{13}= & L U c g q_{A} v w-L U c q_{M} v w \\
& +L U f q_{M} w^{2}+L q_{A} s v^{2}+U c^{2} g z+f s z,
\end{aligned}
$$

Note that if $g q_{A}>q_{M}, \Delta_{13}>0$.

$$
\begin{aligned}
S_{13}= & \frac{N_{S_{13}}}{\Delta_{13}}, \quad A_{13}=\frac{N_{A_{13}}}{\Delta_{13}}, \quad M_{13}=\frac{N_{M_{13}}}{\Delta_{13}}, \\
N_{S_{13}}= & \left(c g q_{A} s v-c g k_{M} q_{A} v-c^{2} g k_{S}\right. \\
& +c^{2} g z+c k_{A} q_{M} w+c m_{A} q_{M} w \\
& \left.-f k_{M} q_{M} w+f q_{M} s w\right) L U^{2} \\
& +\left(f s z-k_{A} q_{A} s v-m_{A} q_{A} s v-f k_{S} s\right) L, \\
N_{A_{13}}= & L\left[U \left(c g k_{S} w-c g w z\right.\right. \\
& -k_{A} q_{M} w^{2}+k_{M} q_{M} v w-m_{A} q_{M} w^{2} \\
& \left.\left.-q_{M} s v w\right)+k_{S} s v-s v z\right] \\
& +U c g\left(s z-k_{M} z\right)-k_{A} s z-m_{A} s z, \\
N_{M_{13}}= & \left(k_{A} q_{A} v w-k_{M} q_{A} v^{2}\right. \\
& +m_{A} q_{A} v w+q_{A} s v^{2}-c k_{S} v+c v z \\
& \left.+f k_{S} w-f w z\right) L U \\
& +\left(c k_{A} z+c m_{A} z-f k_{M} z+f s z\right) U .
\end{aligned}
$$

The equilibrium $P_{13}$ is feasible if and only if the populations are nonnegative, i.e. either one of the following sets of alternative inequalities hold:

$$
\begin{aligned}
N_{S_{13}} \geq 0, & N_{A_{13}} \geq 0, \\
N_{M_{13}} \geq 0, & \Delta_{13}>0 ; \\
N_{S_{13}} \leq 0, & N_{A_{13}} \leq 0, \\
N_{M_{13}} \leq 0, & \Delta_{13}<0 .
\end{aligned}
$$

Finally, we find $P_{14}=\left(0, E_{14}, A_{14}, M_{14}\right)$. Letting

$$
\begin{aligned}
\Delta_{14}= & K U a b c e g-K U a b c h \\
& +K U b^{2} f h+K a^{2} e s+U c^{2} g r+f r s,
\end{aligned}
$$

we have

$$
\begin{aligned}
E_{14}= & \frac{N_{E_{14}}}{\Delta_{14}}, \quad A_{14}=\frac{N_{A_{14}}}{\Delta_{14}}, \quad M_{14}=\frac{N_{M_{14}}}{\Delta_{14}}, \\
N_{E_{14}}= & K\left[U \left(a c e g s-a c e g k_{M}\right.\right. \\
& +b c h k_{A}+b c h m_{A}-b f h k_{M} \\
& \left.+b f h s-c^{2} g k_{E}+c^{2} g r\right)-a e k_{A} s \\
& \left.-a e m_{A} s-f k_{E} s+f r s\right], \\
N_{A_{14}}= & K U\left(a b h k_{M}-a b h s\right. \\
& -b^{2} h k_{A}-b^{2} h m_{A}-b c g r
\end{aligned}
$$

$$
\begin{aligned}
& \left.+b c g k_{E}\right)-U c g k_{M} r+U c g r s \\
& +\operatorname{Kas}\left(k_{E}-r\right)-k_{A} r s-m_{A} r s, \\
& N_{M_{14}}=U\left(K a^{2} e k_{M}-K a^{2} e s\right. \\
& -K a b e k_{A}-K_{a b e m}+K_{a c k} \\
& -K a c r-K b f k_{E}+K b f r \\
& \left.-c k_{A} r-c m_{A} r+f k_{M} r-f r s\right) .
\end{aligned}
$$

Feasibility is ensured by either one of the following sets of alternative inequalities hold:

$$
\begin{aligned}
N_{S_{14}} \geq 0, \quad N_{A_{14}} \geq 0, \\
N_{M_{14}} \geq 0, \quad \Delta_{14}>0 ; \\
N_{S_{14}} \leq 0, \quad N_{A_{14}} \leq 0, \\
N_{M_{14}} \leq 0, \quad \Delta_{14}<0 .
\end{aligned}
$$

\section{A.2. Stability}

\section{A.2.1. Equilibrium $P_{11}$}

At $P_{11}$ one eigenvalue factorizes, $J_{3,3}\left(P_{11}\right)=g c M_{11}-a E_{11}-v S_{11}-$ $\left(m_{A}+k_{A}\right)$. We need to apply the Routh-Hurwitz conditions to the remaining minor $J_{[1,2,4 ; 1,2,4]}$, namely

$$
\left[\begin{array}{ccc}
-\frac{z}{L} S_{11} & u q_{E} S_{11} & w q_{M} S_{11} \\
-u E_{11} & -\frac{r}{K} E_{11} & h b E_{11} \\
-w M_{11} & -b M_{11} & -\frac{s}{U} M_{11}
\end{array}\right]
$$

Clearly, the trace is negative. The remaining conditions entail the use of the sum $M_{2}^{(11)}$ of the principal minors of order two,

$$
\begin{aligned}
M_{2}^{(11)}= & {\left[\frac{r}{K} \frac{s}{U} E_{11} M_{11}+h b^{2} E_{11} M_{11}\right] } \\
& +\left[\frac{z}{L} \frac{s}{U} S_{11} M_{11}+w^{2} q_{M} S_{11} M_{11}\right] \\
& +\left[\frac{z}{L} \frac{r}{K} S_{11} E_{11}+u^{2} q_{E} S_{11} E_{11}\right]
\end{aligned}
$$

For the determinant, we find

$$
\begin{aligned}
& \operatorname{det}\left(J_{[1,2,4 ; 1,2,4]}\right) \\
& =-\frac{M_{11} E_{11} S_{11}}{L K U}\left[r\left(L U q_{M} w^{2}+s z\right)\right. \\
& \quad+\left(u L\left(b w\left(h q_{E}-q_{M}\right) U+q_{E} s u\right)\right. \\
& \left.\left.\quad+z U b^{2} h\right) K\right]<0
\end{aligned}
$$

which is implied by

$h>\frac{q_{M}}{q_{E}}$.

The last condition is

$\operatorname{tr}\left(J_{[1,2,4 ; 1,2,4]}\right) M_{2}^{(11)}<\operatorname{det}\left(J_{[1,2,4 ; 1,2,4]}\right)$.

Extensively, it can be written as

$$
\begin{aligned}
& S_{11} E_{11} M_{11}\left[\left(\left(b w\left(h q_{E}-q_{M}\right) U+q_{E} s u\right) u L\right.\right. \\
& \left.\left.\quad+z U b^{2} h\right) K+r\left(L U q_{M} w^{2}+s z\right)\right] \\
& \quad<\frac{1}{L K U}\left\{\left[L U \left(\left(M_{11} b^{2} h+S_{11} q_{E} u^{2}\right) E_{11}\right.\right.\right. \\
& \left.\left.\quad+w^{2} q_{M} S_{11} M_{11}\right)+M_{11} S_{11} s z\right] K \\
& \quad+E_{11} r\left(L M_{11} s+S_{11} U z\right)\left[\left(L M_{11} s\right.\right. \\
& \left.\left.\left.\quad+S_{11} U z\right) K+r E_{11} L U\right]\right\}
\end{aligned}
$$

The stability conditions are (A.6), (A.8), which is implied by (A.7) and

$g c M_{11}<a E_{11}+v S_{11}+m_{A}+k_{A}$, 


\section{A.2.2. Equilibrium $P_{12}$}

At $P_{12}$ the explicit eigenvalues are $v q_{A} A_{12}+w q_{M} M_{12}+z-k_{S}$ and $e a A_{12}+h b M_{12}+r-k_{E}$. The Routh-Hurwitz conditions on the remaining minor, $J_{[3,4 ; 3,4]}$ are unconditionally satisfied,

$$
\begin{aligned}
\operatorname{tr}\left(J_{[3,4 ; 3,4]}\right)= & -f A_{12}-\frac{s}{U} M_{12}<0, \\
\operatorname{det}\left(J_{[3,4 ; 3,4]}\right)= & f \frac{s}{U} A_{12} M_{12} \\
& +c^{2} g A_{12} M_{12}>0
\end{aligned}
$$

so that the stability conditions are:

$v q_{A} A_{12}+w q_{M} M_{12}+z<k_{S}$,

$e a A_{12}+h b M_{12}+r<k_{E}$.

In the particular case of no insecticide use, these conditions cannot be satisfied, and this point becomes unconditionally unstable.

\section{A.2.3. Equilibrium $P_{13}$}

The only eigenvalue explicitly known at $P_{13}$ is $J_{2,2}\left(P_{13}\right)=e a A_{13}+$ $h b M_{13}-u S_{13}+r-k_{E}$. We then need to apply the Routh-Hurwitz conditions to the remaining minor $J_{[1,3,4 ; 1,3,4]}$. The trace is negative,

$$
\begin{gathered}
\operatorname{tr}\left(J_{[1,3,4 ; 1,3,4]}\right)=-\frac{z}{L} S_{13} \\
-f A_{13}-\frac{s}{U} M_{13}<0 .
\end{gathered}
$$

For the determinant we need

$$
\begin{aligned}
\operatorname{det}\left(J_{[1,3,4 ; 1,3,4]}\right)= & -\left[z\left(U c^{2} g+f s\right)\right. \\
+ & \left(\left(v\left(g q_{A}-q_{M}\right) c+q_{M} f w\right) w U\right. \\
& \left.\left.+q_{A} s v^{2}\right) L\right] \frac{S_{13} M_{13} A_{13}}{L U}<0
\end{aligned}
$$

which is ensured by

$g>\frac{q_{M}}{q_{A}}$.

Further, we find

$$
\begin{aligned}
M_{2}^{(13)}= & {\left[f \frac{s}{U}+g c^{2}\right] A_{13} M_{13} } \\
& +\left[\frac{z}{L} \frac{s}{U}+w^{2} q_{M}\right] S_{13} M_{13} \\
& +\left[\frac{z}{L} f+v^{2} q_{A}\right] S_{13} A_{13}
\end{aligned}
$$

The final condition to be satisfied is

$\operatorname{tr}\left(J_{[1,3,4 ; 1,3,4]}\right) M_{2}^{(13)}<\operatorname{det}\left(J_{[1,3,4 ; 1,3,4]}\right)$

which explicitly become

$$
\begin{aligned}
& M_{13} A_{13} S_{13}\left\{\left[\left(v\left(g q_{A}-q_{M}\right) c+q_{M} f w\right) w U\right.\right. \\
& \left.\left.\quad+q_{A} s v^{2}\right] L+z\left(U c^{2} g+f s\right)\right\} \\
& +\frac{1}{L U}\left\{\left(A_{13} U f+s M_{13}\right) L\right. \\
& +z S_{13} U\left[\left(\left(\left(M_{13} c^{2} g+S_{13} q_{A} v^{2}\right) A_{13}\right.\right.\right. \\
& \left.\left.\left.+q_{M} M_{13} S_{13} w^{2}\right) U+A_{13} M_{13} f s\right) L\right] \\
& \left.\quad+S_{13} z\left(A_{13} U f+s M_{13}\right)\right\}>0 .
\end{aligned}
$$

Also, negativity of the known eigenvalue must be ensured:

$e a A_{13}+h b M_{13}+r<u S_{13}+k_{E}$.

\section{A.2.4. Equilibrium $P_{14}$}

Similar considerations hold for $P_{14}$, where the known eigenvalue is $J_{1,1}\left(P_{14}\right)=u q_{E} E_{14}+v q_{A} A_{14}+w q_{M} M_{14}+z-k_{S}$. The remaining minor reads

$$
J_{[2,3,4 ; 2,3,4]}=\left[\begin{array}{ccc}
-\frac{r}{K} E_{14} & e a E_{14} & h b E_{14} \\
-a A_{14} & -f A_{14} & g c A_{14} \\
-b M_{14} & -c M_{14} & -\frac{s}{U} M_{14}
\end{array}\right]
$$

so that the trace is seen to be negative. The condition for the determinant instead is

$$
\begin{aligned}
\operatorname{det}\left(J_{[2,3,4 ; 2,3,4]}\right)= & -\left[r\left(U c^{2} g+f s\right)\right. \\
+ & (b U(c(e g-h) a+b f h) \\
& \left.\left.+s a^{2} e\right) K\right] \frac{A_{14} M_{14} E_{14}}{K U}<0
\end{aligned}
$$

and is negative if

$g>\frac{h}{e}$.

Moreover, here

$$
\begin{aligned}
M_{2}^{(14)}= & {\left[\frac{s}{U} f+g c^{2}\right] A_{14} M_{14} } \\
& +\left[\frac{r}{K} \frac{s}{U}+h b^{2}\right] M_{14} E_{14} \\
& +\left[\frac{r}{K} f+e a^{2}\right] E_{14} A_{14} .
\end{aligned}
$$

The final stability condition is then $\operatorname{tr}\left(J_{[2,3,4 ; 2,3,4]}\right) \cdot M_{2}^{(14)}<\operatorname{det}\left(J_{[2,3,4 ; 2,3,4]}\right)$.

Explicitly the stability conditions are

$$
\begin{aligned}
& \left\{\left[(c(e g-h) a+b f h) b U+s a^{2} e\right] K\right. \\
& \left.\quad+r\left(U c^{2} g+f s\right)\right\} E_{14} A_{14} M_{14} \\
& <\frac{1}{K U}\left\{\left[\left(\left(E_{14} a^{2} e+M_{14} c^{2} g\right) A_{14}\right.\right.\right. \\
& \left.\left.\quad+E_{14} M_{14} b^{2} h\right) U+s A_{14} M_{14} f\right] K \\
& \left.\quad+r E_{14}\left(A_{14} U f+s M_{14}\right) K+r E_{14} U\right\} .
\end{aligned}
$$

Negativity of the known eigenvalue also entails:

$$
\begin{aligned}
& u q_{E} E_{14}+v q_{A} A_{14} \\
& \quad+w q_{M} M_{14}+z<k_{S} .
\end{aligned}
$$

This point too is therefore unconditionally unstable, if spraying is not used, or even if it is harmless to spiders.

\section{A.2.5. Equilibrium $P_{15}$}

At coexistence $P_{15}$ the Jacobian $J\left(P_{15}\right)$ can be simplified to the form $\left[\begin{array}{cccc}-\frac{z}{L} S_{15} & u q_{E} S_{15} & v q_{A} S_{15} & w q_{M} S_{15} \\ -u E_{15} & -\frac{r}{K} E_{15} & e a E_{15} & h b E_{15} \\ -v A_{15} & -a A_{15} & -f A_{15} & g c A_{15} \\ -w M_{15} & -b M_{15} & -c M_{15} & -\frac{s}{U} M_{15}\end{array}\right]$

so that the trace is negative. The remaining Routh-Hurwitz conditions are:

$$
\begin{aligned}
& \operatorname{det}\left(J\left(P_{15}\right)\right)>0, \\
& \quad \operatorname{tr}\left(J\left(P_{15}\right)\right) M_{2}^{(15)}<M_{3}^{(15)}, \\
& \quad \operatorname{tr}\left(J\left(P_{15}\right)\right) M_{2}^{(15)} M_{3}^{(15)} \\
& >\left(J\left(P_{15}\right)\right)^{2} \cdot \operatorname{det}\left(J\left(P_{15}\right)\right)+\left(M_{3}^{(15)}\right)^{2}
\end{aligned}
$$

where $M_{3}^{(15)}$ is the sum of the principal minors of order three of the matrix $J\left(P_{15}\right)$. We find

$$
\begin{aligned}
\operatorname{det}\left(J\left(P_{15}\right)\right)= & S_{15} E_{15} A_{15} M_{15}\left\{a c e g q_{E} u w\right. \\
& +a^{2} e q_{M} w^{2}-a b e q_{M} v w-a b h q_{A} v w \\
& +b^{2} h q_{A} v^{2}-b c g q_{A} u v-b c h q_{E} u v \\
& +b f h q_{E} u w+c^{2} g q_{E} u^{2}+a c q_{M} u w \\
& -b f q_{M} u w+\frac{1}{L K U}\left[K a^{2} e s z\right. \\
& +K L\left(a e q_{E} s u v-a q_{A} s u v+f q_{E} s u^{2}\right) \\
& +K U\left(a b c e g z-a b c h z+b^{2} f h z\right) \\
& +L U\left(c g q_{A} r v w-c q_{M} r v w+f q_{M} r w^{2}\right) \\
& \left.\left.++L q_{A} r s v^{2}+U c^{2} g r z+f r s z\right]\right\}>0
\end{aligned}
$$


as well as

$$
\begin{aligned}
M_{2}= & \frac{E_{15} S_{15}\left(K L q_{E} u^{2}+r z\right)}{L K} \\
& +\frac{A_{15} E_{15}\left(K a^{2} e+f r\right)}{K} \\
& +\frac{A_{15} M_{15}\left(U c^{2} g+f s\right)}{U} \\
& +\frac{M_{15} S_{15}\left(L U q_{M} w^{2}+s z\right)}{L U} \\
& +\frac{A_{15} S_{15}\left(L a q_{A} v+f z\right)}{L} \\
& +\frac{E_{15} M_{15}\left(K U b^{2} h+r s\right)}{K U}
\end{aligned}
$$

and

$$
\begin{aligned}
M_{3}= & -\frac{E_{15} A_{15} M_{15}}{K U}\{[(c(e g-h) a+b f h) b U \\
& \left.\left.+s a^{2} e\right] K+r\left(U c^{2} g+f s\right)\right\} \\
& -\frac{A_{15} S_{15} M_{15}}{L U}\left[z\left(U c^{2} g+f s\right)\right. \\
& \left.\left.+\left(w\left(v\left(g q_{A}-q_{M}\right) c+q_{M} f w\right) U+q_{A} s v^{2}\right) L\right)\right] \\
& -\frac{S_{15} E_{15} M_{15}}{L K U}\left\{r\left(L U q_{M} w^{2}+s z\right)\right. \\
& +K\left[u\left(b w\left(h q_{E}-q_{M}\right) U+q_{E} s u\right) L\right. \\
& \left.\left.+z U b^{2} h\right]\right\}-\frac{E_{15} M_{15} S_{15}}{L K}\left\{r\left(L q_{A} v w+c z\right)\right. \\
& \left.+\left[u\left(a e q_{E} w-b q_{A} v+c q_{E} u\right) L+z a b e\right] K\right\}
\end{aligned}
$$

Sufficient conditions for having $M_{3}<0$ are

$$
e g>h, \quad g q_{A}>q_{M}, \quad h q_{E}>q_{M},
$$

$a e q_{E} w+c q_{E} u>b q_{A} v$.

The second condition in (A.19) explicitly is

$$
\begin{aligned}
& \left(\frac{z S_{15}}{L}+\frac{r E_{15}}{K}+f A_{15}+\frac{s M_{15}}{U}\right)\left[\frac{E_{15} S_{15}}{L K}(r z\right. \\
& \left.+q_{E} u^{2} K L\right)+\frac{A_{15} E_{15}}{K}\left(e a^{2} K+r f\right) \\
& +\frac{A_{15} M_{15}}{U}\left(g c^{2} U+s f\right)+\frac{M_{15} S_{15}}{L U}(s z \\
& \left.+L U q_{M} w^{2}\right)+\frac{A_{15} S_{15}}{L}\left(q_{A} v a L+z f\right) \\
& \left.+\frac{E_{15} M_{15}}{K U}\left(h b^{2} K U+r s\right)\right] \\
& >\frac{E_{15} M_{15} A_{15}}{K U}\{[b(c(e g-h) a+b f h) U \\
& \left.\left.++s a^{2} e\right] K+r\left(g c^{2} U+s f\right)\right\} \\
& +\frac{M_{15} S_{15} A_{15}}{L U}\left\{z\left(g c^{2} U+s f\right)\right. \\
& \left.+\left[\left(v\left(g q_{A}-q_{M}\right) c+q_{M} f w\right) w U+q_{A} s v^{2}\right] L\right\} \\
& +\frac{E_{15} M_{15} S_{15}}{L K U}\left\{r\left(L U q_{M} w^{2}+s z\right)\right. \\
& +\left[\left(b w\left(h q_{E}-q_{M}\right) U+q_{E} s u\right) u L\right. \\
& \left.\left.+z_{15} b^{2} h\right] K\right\}+\frac{E_{15} M_{15}}{L K}\left\{r\left(L q_{A} v w+c z\right)\right. \\
& \left.+\left[u\left(a e q_{E} w-b q_{A} v+c q_{E} u\right) L+z a b e\right] K\right\}
\end{aligned}
$$

while the last one, although it could be written as well, is too involved and it is omitted. The stability conditions are (A.20), (A.22) and

$$
\begin{aligned}
& \operatorname{tr}\left(J\left(P_{15}\right)\right) M_{2} M_{3} \\
& \quad>\left(J\left(P_{15}\right)\right)^{2} \operatorname{det}\left(J\left(P_{15}\right)\right)+M_{3}^{2}
\end{aligned}
$$

\section{Appendix B. Bifurcations}

Let us rewrite the model (2.1) in synthetic notation as $x^{\prime}(t)=\mathbf{F}(x)$, where $x^{T}=(S, E, A, M)$ and $\mathbf{F}^{T}=\left(F^{1}, F^{2}, F^{3}, F^{4}\right)$ denotes the right hand side of the system. The analysis of the bifurcations is carried out using Sotomayor's Theorem, Perko (2011) and the notation therein, using subscripts to denote partial derivatives. In particular we will need the following quantities:

$$
\begin{gathered}
F_{S S}^{1}=-2 \frac{z}{L}, \quad F_{S E}^{1}=u q_{E}, \\
F_{S A}^{1}=v q_{A}, \quad F_{S M}^{1}=w q_{M}, \\
F_{S E}^{2}=-u, \quad F_{E E}^{2}=-2 \frac{r}{K}, \\
F_{E A}^{2}=e a, \quad F_{E M}^{2}=h b, \\
F_{S A}^{3}=-v, \quad F_{E A}^{3}=-a, \\
F_{A A}^{3}=-2 f, \quad F_{A M}^{3}=g c, \\
F_{S M}^{4}=-w, \quad F_{E M}^{4}=-b, \\
F_{A M}^{4}=-c, \quad F_{M M}^{4}=-2 \frac{s}{U}
\end{gathered}
$$

and where the second derivatives for all the remaining combinations of the variables are zero. Also, all the third order derivatives vanish, i.e. $F_{X Y W}^{k}=0, k=1, \ldots, 4$ and $X, Y, W \in\{S, E, A, M\}$. This last condition implies that no pitchfork bifurcation is possible. In what follows, the eigenvalues that are negative will not give rise to bifurcations for feasible values of the model parameters and therefore will not be considered.

\section{B.1. Bifurcations at $P_{0}$}

For $P_{0}=(0,0,0,0)$ the Jacobian has four explicit eigenvalues,

$\lambda_{1}=z-k_{S}, \quad \lambda_{2}=r-k_{E}$,

$\lambda_{3}=-k_{A}-m_{A}, \quad \lambda_{4}=s-k_{M}$.

\section{B.1.1. Eigenvalue $\lambda_{1}$}

Take as bifurcation parameter $z$ and let $\hat{z}:=k_{S}$. The right $\mathbf{v}$ and left $\mathbf{w}$ eigenvectors of the Jacobian are $\mathbf{v}=\mathbf{w}=(1,0,0,0)^{T}$. Upon suitable differentiation, in this case we find

$$
\begin{aligned}
\mathbf{F}_{\mathbf{z}} & =\left(\begin{array}{c}
S\left(1-\frac{S}{L}\right) \\
0 \\
0 \\
0
\end{array}\right), \\
D \mathbf{F}_{\mathbf{z}} & =\left[\begin{array}{cccc}
1-2 \frac{S}{L} & 0 & 0 & 0 \\
0 & 0 & 0 & 0 \\
0 & 0 & 0 & 0 \\
0 & 0 & 0 & 0
\end{array}\right]
\end{aligned}
$$

so that $\mathbf{F}_{z}\left(P_{0}, \hat{z}\right)=\mathbf{0}$, for which $\mathbf{w}^{\mathbf{T}} \mathbf{F}_{z}\left(P_{0}, \hat{z}\right)=0$, implying $D \mathbf{F}_{z}\left(P_{0}, \hat{z}\right) \mathbf{v}=$ $(1,0,0,0)^{T}$ and therefore $\mathbf{w}^{\mathbf{T}}\left[D \mathbf{F}_{z}\left(P_{0}, \hat{z}\right) \mathbf{v}\right]=1 \neq 0$. Further, $\mathbf{w}^{T}\left[D^{2} \mathbf{F}\left(P_{0}\right.\right.$, $\hat{z})(\mathbf{v}, \mathbf{v})]=-2 \frac{z}{L} \neq 0$, showing a transcritical bifurcation at the equilibrium point $P_{0}$ for the critical parameter value $z=\hat{z}$, between $P_{0}$ and $P_{1}$ for $z=\hat{z}$.

\section{B.1.2. Eigenvalue $\lambda_{2}$}

Take as bifurcation parameter $r$ and let $\hat{r}:=k_{E}$. The right $\mathbf{v}$ and left $\mathbf{w}$ eigenvectors of the Jacobian are $\mathbf{v}=(0,1,0,0)^{T}, \mathbf{w}=(0,1,0,0)^{T}$. Upon suitable differentiation, in this case we find $\mathbf{F}_{r}\left(P_{0}, \hat{r}\right)=\mathbf{0}$, for which $\mathbf{w}^{\mathbf{T}} \mathbf{F}_{r}\left(P_{0}, \hat{r}\right)=0$, implying $D \mathbf{F}_{r}\left(P_{0}, \hat{r}\right) \mathbf{v}=(0,1,0,0)^{T}$ and therefore $\mathbf{w}^{\mathbf{T}}\left[D \mathbf{F}_{r}\left(P_{0}, \hat{r}\right) \mathbf{v}\right]=1 \neq 0$. Further, $\mathbf{w}^{T}\left[D^{2} \mathbf{F}\left(P_{0}, \hat{r}\right)(\mathbf{v}, \mathbf{v})\right]=-2 \frac{r}{K} \neq 0$, showing a transcritical bifurcation at the equilibrium point $P_{0}$ for the critical parameter value $r=\hat{r}$, between $P_{0}$ and $P_{2}$. 


\section{B.1.3. Eigenvalue $\lambda_{4}$}

Take as bifurcation parameter $s$ and let $\hat{s}:=k_{M}$. The right $\mathbf{v}$ and left $\mathbf{w}$ eigenvectors of the Jacobian are $\mathbf{v}=(0,0,0,1)^{T}, \mathbf{w}=(0,0,0,1)^{T}$. Upon suitable differentiation, in this case we find $\mathbf{F}_{s}\left(P_{0}, \hat{s}\right)=\mathbf{0}$, for which $\mathbf{w}^{\mathbf{T}} \mathbf{F}_{s}\left(P_{0}, \hat{s}\right)=0$, implying $D \mathbf{F}_{s}\left(P_{0}, \hat{s}\right) \mathbf{v}=(0,0,0,1)^{T}$ and therefore $\mathbf{w}^{\mathbf{T}}\left[D \mathbf{F}_{s}\left(P_{0}, \hat{s}\right) \mathbf{v}\right]=1 \neq 0$. Further, $\mathbf{w}^{T}\left[D^{2} \mathbf{F}\left(P_{0}, \hat{s}\right)(\mathbf{v}, \mathbf{v})\right]=-2 \frac{s}{U} \neq 0$, showing a transcritical bifurcation at the equilibrium point $P_{0}$ for the critical parameter value $s=\hat{s}$, between $P_{0}$ and $P_{8}$.

\section{B.2. Bifurcations at $P_{1}$}

For $P_{1}=\left(S_{1}, 0,0,0\right)$ the Jacobian has four explicit eigenvalues,

$\lambda_{1}=-z+k_{S}, \quad \lambda_{2}=-u S_{1}+r-k_{E}$,

$\lambda_{3}=-v S_{1}-k_{A}-m_{A}, \quad \lambda_{4}=-w S_{1}+s-k_{M}$.

\section{B.2.1. Eigenvalue $\lambda_{1}$}

Take as bifurcation parameter $z$ and let $\hat{z}:=k_{S}$. The right $\mathbf{v}$ and left $\mathbf{w}$ eigenvectors of the Jacobian are $\mathbf{v}=(1,0,0,0)^{T}, \mathbf{w}=\left(1, w_{2}, w_{3}, w_{4}\right)^{T}$. Upon suitable differentiation, in this case we find $\mathbf{F}_{z}\left(P_{1}, \hat{z}\right)=\mathbf{0}$, for which $\mathbf{w}^{\mathbf{T}} \mathbf{F}_{z}\left(P_{1}, \hat{z}\right)=0$, implying $D \mathbf{F}_{z}\left(P_{1}, \hat{z}\right) \mathbf{v}=(1,0,0,0)^{T}$ and therefore $\mathbf{w}^{\mathbf{T}}\left[D \mathbf{F}_{z}\left(P_{1}, \hat{z}\right) \mathbf{v}\right]=1 \neq 0$. Further, $\mathbf{w}^{T}\left[D^{2} \mathbf{F}\left(P_{1}, \hat{z}\right)(\mathbf{v}, \mathbf{v})\right]=-2 \frac{z}{L} \neq 0$, showing a transcritical bifurcation at the equilibrium point $P_{1}$ for the critical parameter value $z=\hat{z}$, between $P_{1}$ and $P_{0}$ as $z$ crosses the critical threshold $\hat{z}$ from above.

\section{B.2.2. Eigenvalue $\lambda_{2}$}

Take as bifurcation parameter $r$ and let $\hat{r}:=\frac{u\left(z-k_{S}\right) L}{z}+k_{E}$. The right $\mathbf{v}$ and left $\mathbf{w}$ eigenvectors of the Jacobian are $\mathbf{v}=\left(-\frac{{ }^{z} u q_{E} S_{1}}{k_{S}-z}, 1,0,0\right)^{T}, \mathbf{w}=$ $(0,1,0,0)^{T}$. Upon suitable differentiation, in this case we find $\mathbf{F}_{r}\left(P_{1}, \hat{r}\right)=$ $\mathbf{0}$, for which $\mathbf{w}^{\mathbf{T}} \mathbf{F}_{r}\left(P_{1}, \hat{r}\right)=0$, implying $D \mathbf{F}_{r}\left(P_{1}, \hat{r}\right) \mathbf{v}=(0,1,0,0)^{T}$ and therefore

$\mathbf{w}^{\mathbf{T}}\left[D \mathbf{F}_{r}\left(P_{1}, \hat{r}\right) \mathbf{v}\right]=1 \neq 0$. Further, $\mathbf{w}^{T}\left[D^{2} \mathbf{F}\left(P_{1}, \hat{r}\right)(\mathbf{v}, \mathbf{v})\right]=-2\left(\frac{u^{2} q_{E} S_{1}}{k_{S}-z}+\right.$ $\left.\frac{r}{K}\right) \neq 0$, showing a transcritical bifurcation for which the equilibrium point $P_{1}$ becomes $P_{3}$, as the bifurcation parameter crosses the critical value $r=\hat{r}$ from below.

\section{B.2.3. Eigenvalue $\lambda_{4}$}

Take as bifurcation parameter $s$ and let $\hat{s}:=k_{M}+w S_{1}$. The right $\mathbf{v}$ and left $\mathbf{w}$ eigenvectors of the Jacobian are $\mathbf{v}=\left(\frac{w q_{M} L}{z}, 0,0,1\right)^{T}, \mathbf{w}=$ $(0,0,0,1)^{T}$. Upon suitable differentiation, in this case we find $\mathbf{F}_{s}\left(P_{1}, \hat{s}\right)=$ $\mathbf{0}$, for which $\mathbf{w}^{\mathbf{T}} \mathbf{F}_{s}\left(P_{1}, \hat{s}\right)=0$, implying $D \mathbf{F}_{s}\left(P_{1}, \hat{s}\right) \mathbf{v}=(0,0,0,1)^{T}$ and therefore $\mathbf{w}^{\mathbf{T}}\left[D \mathbf{F}_{s}\left(P_{1}, \hat{s}\right) \mathbf{v}\right]=1 \neq 0$. Further, $\mathbf{w}^{T}\left[D^{2} \mathbf{F}\left(P_{1}, \hat{s}\right)(\mathbf{v}, \mathbf{v})\right]=$ $-2\left(\frac{s}{U}+\frac{w^{2} q_{M} L}{z}\right) \neq 0$, showing a transcritical bifurcation at the equilibrium point ${ }^{z} P_{0}$ whenever $s$ increases past the critical value $\hat{s}$, between $P_{1}$ and $P_{9}$.

\section{B.3. Bifurcations at $P_{2}$}

For $P_{2}=\left(0, E_{2}, 0,0\right)$ the Jacobian has four explicit eigenvalues,

$\lambda_{1}=u q_{E} E_{2}+z-k_{S}, \quad \lambda_{2}=k_{E}-r$,

$\lambda_{3}=-a E_{2}-k_{A}-m_{A}, \quad \lambda_{4}=s-b E_{2}-k_{M}$.

\section{B.3.1. Eigenvalue $\lambda_{1}$}

Take as bifurcation parameter $z$ and let $\hat{z}:=k_{S}-u q_{E} E_{2}$, feasible for $k_{S}>u q_{E} E_{2}$. The right $\mathbf{v}$ and left $\mathbf{w}$ eigenvectors of the Jacobian are $\mathbf{v}=\left(-\frac{r}{u K}, 1,0,0\right)^{T}, \mathbf{w}=(1,0,0,0)^{T}$. Upon suitable differentiation, in this case we find $\mathbf{F}_{z}\left(P_{2}, \hat{z}\right)=\mathbf{0}$, for which $\mathbf{w}^{\mathbf{T}} \mathbf{F}_{z}\left(P_{2}, \hat{z}\right)=0$, implying $D \mathbf{F}_{z}\left(P_{2}, \hat{z}\right) \mathbf{v}=\left(-\frac{r}{u K}, 0,0,0\right)^{T}$ and therefore $\mathbf{w}^{\mathbf{T}}\left[D \mathbf{F}_{z}\left(P_{2}, \hat{z}\right) \mathbf{v}\right]=-\frac{r}{u K} \neq 0$. Further, $\mathbf{w}^{T}\left[D^{2} \mathbf{F}\left(P_{2}, \hat{z}\right)(\mathbf{v}, \mathbf{v})\right]=-2\left(\frac{z}{L}\left(\frac{r}{u K}\right)^{2}+q_{E} \frac{r}{K}\right) \neq 0$, showing a transcritical bifurcation at the equilibrium point $P_{2}$ with $P_{3}$ as the bifurcation parameter crosses the critical threshold $z=\hat{z}$ from below.

\section{B.3.2. Eigenvalue $\lambda_{2}$}

Take as bifurcation parameter $r$ and let $\hat{r}:=k_{E}$. The right $\mathbf{v}$ and left $\mathbf{w}$ eigenvectors of the Jacobian are $\mathbf{v}=(0,1,0,0)^{T}, \mathbf{w}=\left(w_{1}, 1, w_{3}, w_{4}\right)^{T}$. Upon suitable differentiation, in this case we find $\mathbf{F}_{r}\left(P_{2}, \hat{r}\right)=\mathbf{0}$, for which $\mathbf{w}^{\mathbf{T}} \mathbf{F}_{r}\left(P_{2}, \hat{r}\right)=0$, implying $D \mathbf{F}_{r}\left(P_{2}, \hat{r}\right) \mathbf{v}=(0,1,0,0)^{T}$ and therefore $\mathbf{w}^{\mathbf{T}}\left[D \mathbf{F}_{r}\left(P_{2}, \hat{r}\right) \mathbf{v}\right]=1 \neq 0$. Further, $\mathbf{w}^{T}\left[D^{2} \mathbf{F}\left(P_{2}, \hat{r}\right)(\mathbf{v}, \mathbf{v})\right]=-2 \frac{r}{K} \neq 0$, showing a transcritical bifurcation at the equilibrium point $P_{2}$ for the critical parameter value $r=\hat{r}$, between $P_{2}$ and $P_{0}$.

\section{B.3.3. Eigenvalue $\lambda_{4}$}

Take as bifurcation parameter $s$ and let $\hat{s}:=k_{M}+b E_{2}$. The right $\mathbf{v}$ and left $\mathbf{w}$ eigenvectors of the Jacobian are $\mathbf{v}=\left(0,1,0, \frac{r}{h b K}\right)^{T}, \mathbf{w}=$ $(0,0,0,1)^{T}$. Upon suitable differentiation, in this case we find $\mathbf{F}_{s}\left(P_{2}, \hat{s}\right)=$ $\mathbf{0}$, for which $\mathbf{w}^{\mathbf{T}} \mathbf{F}_{s}\left(P_{2}, \hat{s}\right)=0$, implying $D \mathbf{F}_{s}\left(P_{2}, \hat{s}\right) \mathbf{v}=\left(0,0,0, \frac{r}{h b K}\right)^{T}$ and therefore $\mathbf{w}^{\mathbf{T}}\left[D \mathbf{F}_{s}\left(P_{2}, \hat{s}\right) \mathbf{v}\right]=\frac{r}{h b K} \neq 0$. Further, $\mathbf{w}^{T}\left[D^{2} \mathbf{F}\left(P_{2}, \hat{s}\right)(\mathbf{v}, \mathbf{v})\right]=$ $-2\left(\frac{s}{U} \frac{r^{2}}{h^{2} b^{2} K^{2}}+\frac{r}{h b K}\right) \neq 0$, showing a transcritical bifurcation at the equilibrium point $P_{2}$ crossing from below the critical parameter value $s=\hat{s}$, between $P_{2}$ and $P_{10}$.

\section{B.4. Bifurcations at $P_{3}$}

For $P_{3}=\left(S_{3}, E_{3}, 0,0\right)$ the Jacobian has two explicit eigenvalues,

$\lambda_{1}=-a E_{3}-v S_{3}-k_{A}-m_{A}$,

$\lambda_{2}=-b E_{3}+s-w S_{3}-k_{M}$.

\section{B.4.1. Eigenvalue $\lambda_{2}$}

Take as bifurcation parameter $s$ and let $\hat{s}:=b E_{3}+w S_{3}+k_{M}$. The right $\mathbf{v}$ and left $\mathbf{w}$ eigenvectors of the Jacobian are $\mathbf{v}=\left(v_{1}, v_{2}, 0,1\right)^{T}, \mathbf{w}=$ $(0,0,0,1)^{T}$. Upon suitable differentiation, in this case we find $\mathbf{F}_{s}\left(P_{3}, \hat{s}\right)=$ $\mathbf{0}$, for which $\mathbf{w}^{\mathbf{T}} \mathbf{F}_{s}\left(P_{3}, \hat{s}\right)=0$, implying $D \mathbf{F}_{s}\left(P_{3}, \hat{s}\right) \mathbf{v}=(0,0,0,1)^{T}$ and therefore $\mathbf{w}^{\mathbf{T}}\left[D \mathbf{F}_{s}\left(P_{3}, \hat{s}\right) \mathbf{v}\right]=1 \neq 0$. Now if $\mathbf{w}^{T}\left[D^{2} \mathbf{F}\left(P_{3}, \hat{s}\right)(\mathbf{v}, \mathbf{v})\right]=2\left(w v_{1}+\right.$ $\left.b v_{2}+s U^{-1}\right) \neq 0$ there is a transcritical bifurcation for the critical parameter value $s=\hat{s}$, between $P_{3}$ and $P_{11}$.

\section{B.5. Bifurcations at $P_{8}$}

For $P_{8}=\left(0,0,0, M_{8}\right)$ the Jacobian has four explicit eigenvalues,

$\lambda_{1}=w q_{M} M_{8}+z-k_{S}, \quad \lambda_{2}=h b M_{8}+r-k_{E}$,

$\lambda_{3}=g c M_{8}-m_{A}-k_{A}, \quad \lambda_{4}=k_{M}-s$.

\section{B.5.1. Eigenvalue $\lambda_{1}$}

Take as bifurcation parameter $z$ and let $\hat{z}:=k_{S}-w q_{M} M_{8}$, feasible for $k_{S}>w q_{M} M_{8}$. The right $\mathbf{v}$ and left $\mathbf{w}$ eigenvectors of the Jacobian are $\mathbf{v}=\left(1,0,0,-\frac{w U}{s}\right)^{T}, \mathbf{w}=(1,0,0,0)^{T}$. Upon suitable differentiation, in this case we find $\mathbf{F}_{z}\left(P_{8}, \hat{z}\right)=\mathbf{0}$, for which $\mathbf{w}^{\mathbf{T}} \mathbf{F}_{z}\left(P_{8}, \hat{z}\right)=0$, implying $D \mathbf{F}_{z}\left(P_{8}, \hat{z}\right) \mathbf{v}=(1,0,0,0)^{T}$ and therefore $\mathbf{w}^{\mathbf{T}}\left[D \mathbf{F}_{z}\left(P_{8}, \hat{z}\right) \mathbf{v}\right]=1 \neq 0$. Further, $\mathbf{w}^{T}\left[D^{2} \mathbf{F}\left(P_{8}, \hat{z}\right)(\mathbf{v}, \mathbf{v})\right]=-2\left(\frac{z}{L}+w^{2} \frac{L U}{s}\right) \neq 0$, showing a transcritical bifurcation for the critical parameter value $z=\hat{z}$, between $P_{8}$ and $P_{9}$.

\section{B.5.2. Eigenvalue $\lambda_{2}$}

Take as bifurcation parameter $r$ and let $\hat{r}:=k_{E}-h b M_{8}$, feasible for $k_{E}>h b M_{8}$. The right $\mathbf{v}$ and left $\mathbf{w}$ eigenvectors of the Jacobian are $\mathbf{v}=\left(0,1,0,-\frac{b U}{s}\right)^{T}, \mathbf{w}=(0,1,0,0)^{T}$. Upon suitable differentiation, in this case we find $\mathbf{F}_{r}\left(P_{8}, \hat{r}\right)=\mathbf{0}$, for which $\mathbf{w}^{\mathbf{T}} \mathbf{F}_{r}\left(P_{8}, \hat{r}\right)=0$, implying $D \mathbf{F}_{r}\left(P_{8}, \hat{r}\right) \mathbf{v}=(0,1,0,0)^{T}$ and therefore $\mathbf{w}^{\mathbf{T}}\left[D \mathbf{F}_{r}\left(P_{8}, \hat{r}\right) \mathbf{v}\right]=1 \neq 0$. Further, $\mathbf{w}^{T}\left[D^{2} \mathbf{F}\left(P_{8}, \hat{r}\right)(\mathbf{v}, \mathbf{v})\right]=-2\left(\frac{r}{K}+\frac{h b^{2} U}{s}\right) \neq 0$, showing a transcritical bifurcation at the equilibrium point $P_{8}$ for the critical parameter value $r=\hat{r}$, between $P_{8}$ and $P_{10}$. 


\section{B.5.3. Eigenvalue $\lambda_{3}$}

Taking as bifurcation parameter $m_{A}$ and letting $\hat{m_{A}}:=g c M_{8}-k_{A}$ we find that this threshold is feasible for $g c M_{8}>k_{A}$. But the threshold for the parameter values used, is negative, thus no feasible bifurcation can occur in terms of $m_{A}$.

However, by choosing $c=\hat{c}:=\frac{m_{A}+k_{A}}{g M_{8}}$, the threshold value is acceptable, namely $\hat{c}=117.8508$. The right $\mathbf{v}$ and left $\mathbf{w}$ eigenvectors of the Jacobian are $\mathbf{v}=\left(0,0,1,-\frac{c U}{s}\right)^{T}, \mathbf{w}=(0,0,1,0)^{T}$. Upon suitable differentiation, in this case we find $\mathbf{F}_{c}\left(P_{8}, \hat{c}\right)=\mathbf{0}$, for which $\mathbf{w}^{\mathbf{T}} \mathbf{F}_{c}\left(P_{8}, \hat{c}\right)=$ 0 , implying $D \mathbf{F}_{c}\left(P_{8}, \hat{c}\right) \mathbf{v}=\left(0,0, g \frac{U\left(s-k_{M}\right)}{s},-\frac{U\left(s-k_{M}\right)}{s}\right)^{T}$ and therefore $\mathbf{w}^{\mathbf{T}}\left[D \mathbf{F}_{c}\left(P_{8}, \hat{c}\right) \mathbf{v}\right]=g \frac{U\left(s-k_{M}\right)}{s} \neq 0$. Further, $\mathbf{w}^{T}\left[D^{2} \mathbf{F}\left(P_{8}, \hat{c}\right)(\mathbf{v}, \mathbf{v})\right]=-2 f-$ $2 g c^{2} \frac{U}{s} \neq 0$, showing a transcritical bifurcation for the critical parameter value $c=\hat{c}$ between $P_{8}$ and $P_{12}$.

\section{B.5.4. Eigenvalue $\lambda_{4}$}

Take as bifurcation parameter $s$ and let $\hat{s}:=k_{M}$. The right $\mathbf{v}$ and left $\mathbf{w}$ eigenvectors of the Jacobian are $\mathbf{v}=(0,0,0,1)^{T}, \mathbf{w}=\left(w_{1}, w_{2}, w_{3}, 1\right)^{T}$. Upon suitable differentiation, in this case we find $\mathbf{F}_{s}\left(P_{8}, \hat{s}\right)=\mathbf{0}$, for which $\mathbf{w}^{\mathbf{T}} \mathbf{F}_{s}\left(P_{8}, \hat{s}\right)=0$, implying $D \mathbf{F}_{s}\left(P_{8}, \hat{s}\right) \mathbf{v}=(0,0,0,1)^{T}$ and therefore $\mathbf{w}^{\mathbf{T}}\left[D \mathbf{F}_{s}\left(P_{8}, \hat{s}\right) \mathbf{v}\right]=1 \neq 0$. Further, $\mathbf{w}^{T}\left[D^{2} \mathbf{F}\left(P_{8}, \hat{s}\right)(\mathbf{v}, \mathbf{v})\right]=-2 \frac{s}{U} \neq 0$, showing a transcritical bifurcation at the equilibrium point $P_{0}$ for the critical parameter value $s=\hat{s}$, between $P_{8}$ and $P_{0}$.

\section{B.6. Bifurcations at $P_{9}$}

For $P_{9}=\left(S_{9}, 0,0, M_{9}\right)$ the Jacobian has two explicit eigenvalues,

$\lambda_{1}=h b M_{9}+r-k_{E}-u S_{9}$,

$\lambda_{2}=g c M_{9}-m_{A}-k_{A}-v S_{9}$.

\section{B.6.1. Eigenvalue $\lambda_{1}$}

Take as bifurcation parameter $r$ and let $\hat{r}:=k_{E}+u S_{9}-h b M_{9}$, feasible for $k_{E}+u S_{9}>h b M_{9}$. The right $\mathbf{v}$ and left $\mathbf{w}$ eigenvectors of the Jacobian are $\mathbf{v}=\left(v_{1}, 1,0, v_{4}\right)^{T}, \mathbf{w}=(0,1,0,0)^{T}$. Upon suitable differentiation, in this case we find $\mathbf{F}_{r}\left(P_{9}, \hat{r}\right)=\mathbf{0}$, for which $\mathbf{w}^{\mathbf{T}} \mathbf{F}_{r}\left(P_{9}, \hat{r}\right)=0$, implying $D \mathbf{F}_{r}\left(P_{9}, \hat{r}\right) \mathbf{v}=(0,1,0,0)^{T}$ and therefore $\mathbf{w}^{\mathbf{T}}\left[D \mathbf{F}_{r}\left(P_{9}, \hat{r}\right) \mathbf{v}\right]=1 \neq 0$. Further, $\mathbf{w}^{T}\left[D^{2} \mathbf{F}\left(P_{9}, \hat{r}\right)(\mathbf{v}, \mathbf{v})\right]=-2 u v_{1}-2 r K^{-1}+2 h b v_{4}$. Now if $u v_{1}+r K^{-1} \neq h b v_{4}$ a transcritical bifurcation arises for the critical parameter value $r=\hat{r}$, between $P_{9}$ and $P_{11}$.

\section{B.6.2. Eigenvalue $\lambda_{2}$}

Taking as bifurcation parameter $m_{A}$ and letting $\hat{m_{A}}:=g c M_{9}-$ $v S_{9}-k_{A}$, for the parameter values used the threshold is negative, $g c M_{9}<v S_{9}+k_{A}$, thus no feasible bifurcation can occur in terms of $m_{A}$.

However, we can take as bifurcation parameter $c$, for which the threshold value is acceptable, namely $\hat{c}=0.4437$. The right $\mathbf{v}$ and left $\mathbf{w}$ eigenvectors of the Jacobian are $\mathbf{v}=\left(t_{1}, 0,1, t_{4}\right)^{T}, \mathbf{w}=(0,0,1,0)^{T}$. Upon suitable differentiation, in this case we find $\mathbf{F}_{c}\left(P_{9}, \hat{c}\right)=\mathbf{0}$, for which $\mathbf{w}^{\mathbf{T}} \mathbf{F}_{c}\left(P_{9}, \hat{c}\right)=0$, implying $D \mathbf{F}_{c}\left(P_{9}, \hat{m}_{A}\right) \mathbf{v}=\left(0,0, g M_{9},-M_{9}\right)^{T}$ and therefore $\mathbf{w}^{\mathbf{T}}\left[D \mathbf{F}_{c}\left(P_{9}, \hat{c}\right) \mathbf{v}\right]=g M_{9} \neq 0$. Here, $\mathbf{w}^{T}\left[D^{2} \mathbf{F}\left(P_{9}, \hat{m_{A}}\right)(\mathbf{v}, \mathbf{v})\right]=$ $2\left(c g v_{4}-v v_{1}-f\right)$, so that if $\left(c g v_{4}-v v_{1}-f\right) \neq 0$ a transcritical bifurcation occurs for the critical parameter value $c=\hat{c}$ between $P_{9}$ and $P_{13}$.

\section{B.7. Bifurcations at $P_{10}$}

For $P_{10}=\left(0, E_{10}, 0, M_{10}\right)$ the Jacobian has two explicit eigenvalues,

$\lambda_{1}=u q_{E} E_{10}+z+w q_{M} M_{10}-k_{S}$,

$\lambda_{2}=g c M_{10}-m_{A}-k_{A}-a E_{10}$.

\section{B.7.1. Eigenvalue $\lambda_{1}$}

Take as bifurcation parameter $z$ and let $\hat{z}:=k_{S}-u q_{E} E_{10}-w q_{M} M_{10}$, feasible for $k_{S}>u q_{E} E_{10}+w q_{M} M_{10}$. The right $\mathbf{v}$ and left $\mathbf{w}$ eigenvectors of the Jacobian are $\mathbf{v}=\left(1, v_{2}, 0, v_{4}\right)^{T}, \mathbf{w}=(1,0,0,0)^{T}$. Upon suitable differentiation, in this case we find $\mathbf{F}_{z}\left(P_{10}, \hat{z}\right)=\mathbf{0}$, for which $\mathbf{w}^{\mathbf{T}} \mathbf{F}_{z}\left(P_{10}, \hat{r}\right)=$ 0 , implying $D \mathbf{F}_{z}\left(P_{10}, \hat{z}\right) \mathbf{v}=(0,1,0,0)^{T}$ and therefore $\mathbf{w}^{\mathbf{T}}\left[D \mathbf{F}_{z}\left(P_{10}, \hat{r}\right) \mathbf{v}\right]=$ $1 \neq 0$. In this case $\mathbf{w}^{T}\left[D^{2} \mathbf{F}\left(P_{10}, \hat{z}\right)(\mathbf{v}, \mathbf{v})\right]=-2 z L^{-1}+2 u q_{E} v_{2}+2 w q_{M} v_{4}$ so that if $z \neq L\left(u q_{E} v_{2}+w q_{M} v_{4}\right)$ a transcritical bifurcation exists for the critical parameter value $z=\hat{z}$, for $z=\hat{z}$ between $P_{10}$ and $P_{11}$.

\section{B.7.2. Eigenvalue $\lambda_{2}$}

Take as bifurcation parameter $m_{A}$ and let $\hat{m_{A}}:=-k_{A}-a E_{10}+g c M_{10}$, feasible for $g c M_{10}>k_{A}+a E_{10}$. But this threshold is negative, for the parameters chosen. Thus no bifurcation occurs for this choice.

However, we can take as bifurcation parameter $c$. The threshold value is acceptable, namely $\hat{c}=1.4903 e+03$. The right $\mathbf{v}$ and left $\mathbf{w}$ eigenvectors of the Jacobian are $\mathbf{v}=\left(0, v_{2}, 1, v_{4}\right)^{T}, \mathbf{w}=(0,0,1,0)^{T}$. Upon suitable differentiation, in this case we find $\mathbf{F}_{c}\left(P_{10}, \hat{c}\right)=\mathbf{0}$, for which $\mathbf{w}^{\mathbf{T}} \mathbf{F}_{c}\left(P_{10}, \hat{c}\right)=0$, implying $D \mathbf{F}_{c}\left(P_{10}, \hat{c}\right) \mathbf{v}=\left(0,0, g M_{10},-M_{10}\right)^{T}$ and therefore $\mathbf{w}^{\mathbf{T}}\left[D \mathbf{F}_{c}\left(P_{10}, \hat{c}\right) \mathbf{v}\right]=g M_{10} \neq 0$. Then, $\mathbf{w}^{T}\left[D^{2} \mathbf{F}\left(P_{10}, \hat{c}\right)(\mathbf{v}, \mathbf{v})\right]=$ $-2\left(a t_{2}-f+g c t_{4}\right)$, so that if $\left(a t_{2}-f+g c t_{4}\right) \neq 0$ showing a transcritical bifurcation for the critical parameter value $c=\hat{c}$ between $P_{10}$ and $P_{14}$.

\section{B.8. Bifurcations at $P_{11}$}

For $P_{11}=\left(S_{11}, E_{11}, 0, M_{11}\right)$ the Jacobian has one explicit eigenvalue, $\lambda_{1}=g c M_{11}-a E_{11}-v S_{11}-\left(m_{A}+k_{A}\right)$,

\section{B.8.1. Eigenvalue $\lambda_{1}$}

Taking as bifurcation parameter $m_{A}$ and let $\hat{m_{A}}:=g c M_{11}-a E_{11}-$ $v S_{11}-k_{A}$, feasible for $g c M_{11}>a E_{11}+v S_{11}+k_{A}$, it observed however that the threshold is once again negative and no bifurcation occurs.

Instead, use $c$ as bifurcation parameter, with threshold $\hat{c}:=$ $\frac{a E_{11}+v S_{11}+k_{A}}{g M_{11}}=640.6672$. The right $\mathbf{v}$ and left $\mathbf{w}$ eigenvectors of the Jacobian are $\mathbf{v}=\left(v_{1}, v_{2}, 1, v_{4}\right)^{T}, \mathbf{w}=(0,0,1,0)^{T}$. Upon suitable differentiation, in this case we find $\mathbf{F}_{c}\left(P_{11}, \hat{c}\right)=\mathbf{0}$, for which $\mathbf{w}^{\mathbf{T}} \mathbf{F}_{c}\left(P_{11}, \hat{c}\right)=0$, implying $D \mathbf{F}_{c}\left(P_{11}, \hat{c}\right) \mathbf{v}=\left(0,0, g M_{11},-M_{11}\right)^{T}$ and therefore $\mathbf{w}^{\mathbf{T}}\left[D \mathbf{F}_{c}\left(P_{11}, \hat{c}\right) \mathbf{v}\right]$ $=g M_{11} \neq 0$. Further, $\mathbf{w}^{T}\left[D^{2} \mathbf{F}\left(P_{11}, \hat{c}\right)(\mathbf{v}, \mathbf{v})\right]=-2 v v_{1}-2 a v_{2}-2 f+2 c g v_{4}$. Now if $v v_{1}+a v_{2}+f \neq c g v_{4} \neq 0$ we have a transcritical bifurcation as the parameter $c=\hat{c}$ with coexistence $P_{15}$.

Observe further that if

$\operatorname{tr}\left(J_{[3 ; 3]}\right) \cdot M_{2}=\operatorname{det}\left(J_{[3 ; 3]}\right)$

a Hopf bifurcation occurs. Explicitly, it reads

$$
\begin{aligned}
& L K U S_{11} E_{11} M_{11}\left\{r\left(L U q_{M} w^{2}+s z\right)\right. \\
& \quad+\left[\left(b w\left(h q_{E}-q_{M}\right) U+q_{E} s u\right) u L\right. \\
& \left.\left.\quad+z U b^{2} h\right] K\right\}+\left[U \left(\left(M_{11} b^{2} h\right.\right.\right. \\
& \left.+S_{11} q_{E} u^{2}\right) E_{11} \\
& \left.\left.+w^{2} q_{M} S_{11} M_{11}\right) L+M_{11} S_{11} s z\right] K \\
& +E_{11} r\left(L M_{11} s+S_{11} U z\right)\left[\left(L M_{11} s\right.\right. \\
& \left.\left.+S_{11} U z\right) K+r E_{11} L U\right]=0 .
\end{aligned}
$$

Note that for $h q_{M}>q_{E}$ the equality is never satisfied and no Hopf bifurcation can occur.

\section{B.9. Bifurcations at $P_{12}$}

For $P_{12}=\left(0,0, A_{12}, M_{12}\right)$ the Jacobian has two explicit eigenvalues, $\lambda_{1}=v q_{A} A_{12}+w q_{M} M_{12}+z-k_{S}$, $\lambda_{2}=e a A_{12}+h b M_{12}+r-k_{E}$. 


\section{B.9.1. Eigenvalue $\lambda_{1}$}

Take as bifurcation parameter $z$ and let $\hat{z}:=-v q_{A} A_{12}-w q_{M} M_{12}+$ $k_{S}$, feasible for $k_{S}>v q_{A} A_{12}+w q_{M} M_{12}$. The right $\mathbf{v}$ and left $\mathbf{w}$ eigenvectors of the Jacobian are $\mathbf{v}=\left(1,0, v_{3}, v_{4}\right)^{T}, \mathbf{w}=(1,0,0,0)^{T}$. Upon suitable differentiation, in this case we find $\mathbf{F}_{z}\left(P_{12}, \hat{z}\right)=\mathbf{0}$, for which $\mathbf{w}^{\mathbf{T}} \mathbf{F}_{z}\left(P_{12}, \hat{r}\right)=0$, implying $D \mathbf{F}_{z}\left(P_{12}, \hat{z}\right) \mathbf{v}=(0,1,0,0)^{T}$ and therefore $\mathbf{w}^{\mathbf{T}}\left[D \mathbf{F}_{z}\left(P_{12}, \hat{z}\right) \mathbf{v}\right]=1 \neq 0$. Also, $\mathbf{w}^{T}\left[D^{2} \mathbf{F}\left(P_{12}, \hat{z}\right)(\mathbf{v}, \mathbf{v})\right]=$ $-2 z L^{-1}+2 v q_{A} v_{3}+2 w q_{M} v_{4}$. Now if $z L^{-1} \neq v q_{A} v_{3}+w q_{M} v_{4}$ a transcritical bifurcation arises for the critical parameter value $z=\hat{z}$, between $P_{12}$ and $P_{13}$ for $z=\hat{z}$.

\section{B.9.2. Eigenvalue $\lambda_{2}$}

Take as bifurcation parameter $r$ and let $\hat{r}:=k_{E}-e a A_{12}-h b M_{12}$, feasible for $k_{E}>e a A_{12}+h b M_{12}$. The right $\mathbf{v}$ and left $\mathbf{w}$ eigenvectors of the Jacobian are $\mathbf{v}=\left(0,1, v_{3}, v_{4}\right)^{T}, \mathbf{w}=(0,1,0,0)^{T}$. Upon suitable differentiation, in this case we find $\mathbf{F}_{r}\left(P_{12}, \hat{r}\right)=\mathbf{0}$, for which $\mathbf{w}^{\mathbf{T}} \mathbf{F}_{r}\left(P_{12}, \hat{m_{A}}\right)=$ 0 , implying $D \mathbf{F}_{r}\left(P_{12}, \hat{r}\right) \mathbf{v}=(0,0,-1,0)^{T}$ and therefore $\mathbf{w}^{\mathbf{T}}\left[D \mathbf{F}_{r}\left(P_{12}, \hat{r}\right) \mathbf{v}\right]=$ $-1 \neq 0$. Further, $\mathbf{w}^{T}\left[D^{2} \mathbf{F}\left(P_{12}, \hat{r}\right)(\mathbf{v}, \mathbf{v})\right]=-2 a v_{2}-2 f+2 g c v_{4}$. Now if $a v_{2}+f \neq g c v_{4}$ a transcritical bifurcation occurs for the critical parameter value $r=\hat{r}$, between $P_{12}$ and $P_{14}$

\section{B.10. Bifurcations at $P_{13}$}

For $P_{13}=\left(S_{13}, 0, A_{13}, M_{13}\right)$ the Jacobian has one explicit eigenvalue, $\lambda_{1}=e a A_{13}+h b M_{13}-u S_{13}+r-k_{E}$,

\section{B.10.1. Eigenvalue $\lambda_{1}$}

Take as bifurcation parameter $r$ and let $\hat{r}:=-e a A_{13}-h b M_{13}+u S_{13}+$ $k_{E}$, feasible for $u S_{13}+k_{E}>e a A_{13}+h b M_{13}$. The right $\mathbf{v}$ and left $\mathbf{w}$ eigenvectors of the Jacobian are $\mathbf{v}=\left(v_{1}, 1, v_{3}, v_{4}\right)^{T}, \mathbf{w}=(0,1,0,0)^{T}$. Upon suitable differentiation, in this case we find $\mathbf{F}_{r}\left(P_{13}, \hat{r}\right)=\mathbf{0}$, for which $\mathbf{w}^{\mathbf{T}} \mathbf{F}_{r}\left(P_{13}, \hat{r}\right)=0$, implying $D \mathbf{F}_{r}\left(P_{13}, \hat{r}\right) \mathbf{v}=(0,1,0,0)^{T}$ and therefore $\mathbf{w}^{\mathbf{T}}\left[D \mathbf{F}_{r}\left(P_{13}, \hat{r}\right) \mathbf{v}\right]=1 \neq 0$. Further, $\mathbf{w}^{T}\left[D^{2} \mathbf{F}\left(P_{13}, \hat{r}\right)(\mathbf{v}, \mathbf{v})\right]=$ $-2 v_{1}-2 r K^{-1}+2 e a v_{3}+2 h b v_{4}$. if $v_{1}+r K^{-1} \neq e a v_{3}+h b v_{4}$ we have a transcritical bifurcation as the parameter $r=\hat{r}$ the coexistence equilibrium $P_{15}$.

Observe that a Hopf bifurcation arises if

$\operatorname{tr}\left(J_{[2 ; 2]}\right) \cdot M_{2}=\operatorname{det}\left(J_{[2 ; 2]}\right)$

which explicitly is

$$
\begin{aligned}
& L U M_{13} A_{13} S_{13}\left\{z\left(U c^{2} g+f s\right)\right. \\
& \quad+\left[\left(v\left(g q_{A}-q_{M}\right) c+q_{M} f w\right) w U\right. \\
& \left.\left.\quad+q_{A} s v^{2}\right] L\right\}+\left[\left(A_{13} U f+s M_{13}\right) L\right. \\
& +z S_{13} U L\left[\left(M_{13} c^{2} g+S_{13} q_{A} v^{2}\right) A_{13}\right. \\
& \left.\left.+q_{M} M_{13} S_{13} w^{2}\right] U+A_{13} M_{13} f s\right] \\
& \quad+S_{13} z\left(A_{13} U f+s M_{13}\right)=0 .
\end{aligned}
$$

Also, if $g q_{M}>q_{A}$, the equality is never satisfied and no Hopf bifurcation occurs.

\section{B.11. Bifurcations at $P_{14}$}

For $P_{14}=\left(0, E_{14}, A_{14}, M_{14}\right)$ the Jacobian has one explicit eigenvalue, $\lambda_{1}=u q_{E} E_{14}+v q_{A} A_{14}+w q_{M} M_{14}+z-k_{S}$,

\section{B.11.1. Eigenvalue $\lambda_{1}$}

Take as bifurcation parameter $z$ and let $\hat{z}:=k_{S}-u q_{E} E_{14}-v q_{A} A_{14}-$ $w q_{M} M_{14}$, feasible for $k_{S}>u q_{E} E_{14}+v q_{A} A_{14}+w q_{M} M_{14}$. The right $\mathbf{v}$ and left $\mathbf{w}$ eigenvectors of the Jacobian are $\mathbf{v}=\left(1, v_{2}, v_{3}, v_{4}\right)^{T}$, $\mathbf{w}=(1,0,0,0)^{T}$. Upon suitable differentiation, in this case we find $\mathbf{F}_{z}\left(P_{14}, \hat{z}\right)=\mathbf{0}$, for which $\mathbf{w}^{\mathbf{T}} \mathbf{F}_{z}\left(P_{14}, \hat{z}\right)=0$, implying $D \mathbf{F}_{z}\left(P_{14}, \hat{z}\right) \mathbf{v}=$ $(0,1,0,0)^{T}$ and therefore $\mathbf{w}^{\mathbf{T}}\left[D \mathbf{F}_{z}\left(P_{14}, \hat{r}\right) \mathbf{v}\right]=1 \neq 0$. Further, $\mathbf{w}^{T}\left[D^{2} \mathbf{F}\left(P_{14}, \hat{z}\right)(\mathbf{v}, \mathbf{v})\right]=-2 z L^{-1}+2 u q_{E} v_{2}+2 v q_{A} v_{3}+2 w q_{M} v_{4}$. Now if $z L^{-1} \neq u q_{E} v_{2}+v q_{A} v_{3}+w q_{M} v_{4}$ a transcritical bifurcation occurs as the parameter $z=\hat{z}$ with the coexistence point $P_{15}$.

If the equality $\operatorname{tr}\left(J_{[1 ; 1]}\right) \cdot M_{2}^{(13)}=\operatorname{det}\left(J_{[1 ; 1]}\right)$ holds a Hopf bifurcation occurs. Extensively, the condition becomes

$$
\begin{aligned}
0 & =E_{14} A_{14} M_{14}\left\{r\left(U c^{2} g+f s\right)\right. \\
& \left.+\left[(c(e g-h) a+b f h) b U+s a^{2} e\right] K\right\} \\
& +\frac{1}{K U}\left\{\left[\left(\left(E_{14} a^{2} e+M_{14} c^{2} g\right) A_{14}\right.\right.\right. \\
& \left.+\left(E_{14} M_{14} b^{2} h\right) U+s A_{14} M_{14} f\right] K \\
& \left.+r E_{14}\left(A_{14} U f+s M_{14}\right) K+r E_{14} U\right\} .
\end{aligned}
$$

Note that if $e g-h>0$ no Hopf bifurcation occurs.

\section{References}

Arambourg, Y., Pralavorio, R., 1986. Hyponomeutidae Pray oleae. In: Arambourg, Y. (Ed.), Traité d'entomologie oléicole. Conseil Oléicole International, Madrid, Spain, pp. 47-91.

Bartolini, G., Petruccelli, R., 2002. Classification, Origin, Diffusion and History of the Olive. Food and Agriculture organization of the United Nations, Rome, Italy.

Benhadi-Marín, J., Pereira, J.A., Bento, A., Sousa, J.P., Santos, S.A.P., 2016. Biodiversity of spiders in agroecosystems: community structure, conservation and roles as biological control agents. In: Santos, S.A.P. (Ed.), Natural Enemies: Identification, Protection Strategies and Ecological Impacts. Nova Science Publishers, New York, USA, pp. 43-110.

Benhadi-Marín, J., Villa, M., Pereira, L.F., Rodrigues, I., Morente, M., Baptista, P., Pereira, J.A., 2020. A guild-based protocol to target potential natural enemies of Philaenus spumarius (Hemiptera: Aphrophoridae), a vector of Xylella fastidiosa (Xanthomonadaceae): A case study with spiders in the olive grove. Insects 11 (2), 100.

Bento, A.A., 1999. Contribuição para o estabelecimento de um programa de Protecção Integrada contra a traça da oliveira (Prays oleae Bern) em Trás-os-Montes (Ph.D. thesis). Vila Real: Universidade de Trás-os-Montes e Alto Douro, Portugal.

Bento, A., Ilideo, J., Campos, M., Torres, L., 1998. Parasitismo associado à traça da oliveira Prays oleae Bern., em Trás-os-Montes (Nordeste de Portugal). (Parasitism associated to the olive tree moth Prays oleae Bern., in the Trás-os-Montes region (Northeast of Portugal). Bol. San. Veg. Plagas 24, 949-954.

Bento, A., Torres, L., CabanaS, J.E., Pereira, J.A., 2007. A traça da oliveira, Prays oleae (Bernard). pp. 202-209, In: Azevedo J. (Ed) Manual de protecção integrada do olival. Viseu, Portugal.

Bueno, M., 1981. Factores de regulación de las poblaciones de Prays oleae (Bern.). Bol. San. Veg. Plagas. 7, 133-140.

CABI, 2021. https://www.cabi.org/isc/datasheet/43913. (Accessed 25 June 2021).

Campos, M., Ramos, P., 1982. Ageniaspis fuscicollis praysincola Silv. (Hym. Encyrtidae) parásito de Prays oleae Bern. (Lep. Hyponomeutidae) en Granada. (Ageniaspis fuscicollis praysincola Silv. (Hym. Encyrtidae) parasite of Prays oleae Bern. (Lep. Hyponomeutidae) in Granada (Spain). Bol. Asoc. Esp. Entomol. 6, 63-71.

Castex, V., Beniston, M., Calanca, P., Fleury, D., Moreau, J., 2018. Pest management under climate change: The importance of understanding tritrophic relations. Sci. Total Environ. 616-617, 397-407.

Dainese, M., Scheider, G., Krauss, J., Steffan-Dewenter, I.S., 2017. Complementarity among natural enemies enhances pest suppression. Sci. Rep. 7 (8172), http://dx. doi.org/10.1038/s41598-017-08316-z.

DGAV, 2021. https://sifito.dgav.pt/Account/Login?ReturnUrl=\%2F. (Accessed: 17-032021).

Herz, A., Hassan, S.A., Hegazi, E., Nasr, F.N., A, A. Youssef, Khafagi, W.E., Agamy, E., Ksantini, M., Jardak, J.T., Mazomenos, B.E., Konstantopoulou, M.A., Torres, L., Gonçalves, F., Bento, A., Pereira, J.A., 2005. Towards sustainable control of lepidopterous pests in olive cultivation. Gesunde Pflanz. 57, 117-128.

INE, 2021. http://www.ine.pt. (Accessed: 15-7-2021).

Jervis, M.A., Ellers, J., Harvey, J.A., 2008. Resource acquisition, allocation, and utilization in parasitoid reproductive strategies. Annu. Rev. Entomol. 53, 361-385.

Jervis, M.A., Kidd, N.A.C., Fitton, M.G., Huddleston, T., Dawah, H.A., 1993. Flower-visiting by hymenopteran parasitoids. J. Nat. Hist. 27, 67-105.

Marc, P., Canard, A., Ysnel, F., 1999. Spiders (Araneae) useful for pest limitation and bioindication. Agr. Ecosyst. Environ. 74, 229-273. 
Nyffeler, M., Sunderland, K.D., 2003. Composition, abundance and pest control potential of spider communities in agroecosystems: a comparison of European and US studies. Agr. Ecosyst. Environ. 95, 579-612.

Paredes, D., Batuecas, I., Cayuela, L., Campos, M., 2014. Anthocoris nemoralis: a new ally in the conservation biological control of the anthophagous generation of the olive pest Prays oleae. Agroecología 9 (1,2), 79-84.

Pascual, S., Cobos, G., Seris, E., González-Núñez, M., 2010. Effects of processed kaolin on pests and non-target arthropods in a spanish olive grove. J. Pest. Sci. 83, 121-133.

Perko, L., 2011. Differential Equations and Dynamical Systems. Springer, New York, USA.

Pitzer, E.M., Williams, M.T., Vorhees, C.V., 2021. Effects of pyrethroids on brain development and behavior. Deltamethrin. Neurotoxicol. Teratol. 87, 106983.

Pollard, C.P., Griffin, C.T., de Andrade Moral, R., Duffy, C., Chuche, J., Gaffney, M.T., Fealy, R.M., Fealy, R., 2020. phenModel: A temperature-dependent phenology/voltinism model for a herbivorous insect incorporating facultative diapause and budburst. Ecol. Model. 416, 108910.

Riechert, E.S., Lockley, T., 1984. Spiders as biological control agents. Annu. Rev. Entomol. 29, 299-320.

Riechert, S., Maupin, J., 1997. Spider effects on prey: tests for superfluous killing in five web-builders. In: Proceedings of the 17th European Colloquium of Arachnology, Edinburgh, Scotland.
Rossini, L., Rosselló, N.B., Speranza, S., Garone, E., 2021. A general ODE-based model to describe the physiological age structure of ectotherms: Description and application to Drosophila suzukii. Ecol. Model. 456, 109673.

Sharma, A., Shukla, A., Attri, K., Kumar, M., Kumar, P., Suttee, A., Sing, G., Barnwal, R.P., Singla, N., 2020. Global trends in pesticides: a looming threat and viable alternative. Ecotox. Environ. Safe. 201, 110812.

Villa, M., Marrão, R., Mexia, A., Bento, A., Pereira, J.A., 2016a. Are wild flowers and insect honeydews potential food resources for adults of the olive moth, Prays oleae? J. Pest Sci. 90, 185-194. http://dx.doi.org/10.1007/s10340-016-0745-8.

Villa, M., Santos, S.A.P., Mexia, A., Bento, A., Pereira, J.A., 2016b. Ground cover management affects parasitism of Prays oleae (Bernard). Biol. Control 96, 72-77.

Villa, M., Santos, S.A.P., Mexia, A., Bento, A., Pereira, J.A., 2017a. Wild flower resources and insect honeydew are potential food items for Elasmus flabellatus. Agron. Sustain. Dev. (37), 15.

Villa, M., Santos., S.A.P., Pascual, S., Pereira, J.A., 2021. Do non-crop areas and landscape structure influence dispersal and population densities of male olive moth? Bull. Entomol. Res. 111 (1), 73-81.

Villa, M., Santos, S.A.P., Pereira, J.A., 2017b. Análisis de supervivencia de Ageniaspis fuscicollis (Dalman), parasitoide de la polilla del olivo. Tech. Rep., X Congreso Nacional de Entomología Aplicada. Logroño. Spain.

Yefremova, Z.A., Strakhova, I.S., 2010. A review of the species of the genus Elasmus Westwood (Hymenoptera, Eulophidae) from Russia and neighboring countries. Entomol. Rev. 7, 903-926. 